\title{
A Study of Solid/Liquid Interfacial Phenomena in Hypereutectic Al-Si with High Resolution in situ Transmission Electron Microscopy
}

\author{
by \\ Matthew M. Schneider \\ A thesis submitted in partial fulfillment for the degree of \\ Master of Science, Materials Science and Engineering \\ in the \\ Department of Materials Science and Engineering
}

December 2014 


\title{
APPROVAL SHEET
}

This thesis

is submitted in partial fulfillment of the requirements

for the degree of

Master of Science

\section{Matthew M. Schneider}

Author

The thesis has been read and approved by the examining committee:

\author{
James M. Howe
}

Advisor

William A. Soffa

\section{$\underline{\text { Petra Reinke }}$}

Accepted for the School of Engineering and Applied Science:

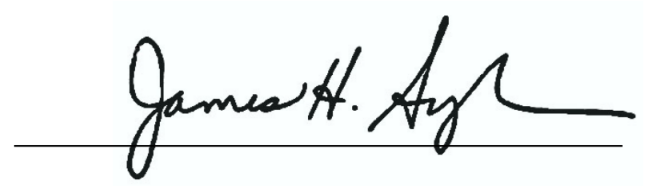

Dean, School of Engineering and Applied Science December 2014 
"Time... is never time at all

You can never ever leave without leaving a piece of youth

And our lives are forever changed

We will never be the same

The more you change the less you feel

Believe, believe in me, believe

That life can change, that you're not stuck in vain

We're not the same, we're different tonight

Tonight, so bright

Tonight"

- Billy Corgan 


\title{
UNIVERSITY OF VIRGINIA
}

\author{
Abstract \\ Department of Materials Science and Engineering \\ Master of Science, Materials Science and Engineering \\ by Matthew M. Schneider
}

Solid-liquid interfaces are extensively encountered in materials processing and applications. The structure and properties of these interfaces affect solidification, wetting behavior, and crystalline growth mechanisms, including epitaxial growth. The exact structural, dynamic, and chemical nature of these interfaces have been historically difficult to study at high resolution due to the fact that one phase is a liquid. Recently, advances in in situ high-resolution transmission electron microscopy experimental methods have progressed to enable the study of solidliquid interfaces in metals with resolution on the atomic scale. Reported results thus far include direct observation of step/ledge growth, the presentation of various crystallographic facets during growth/dissolution, ordering of liquid layers adjacent to crystalline solids, and chemical segregation at interfaces.

This work reports the first observations of a number of new interfacial phenomena associated with the dynamic behavior of the solid/liquid phase boundary at equilibrium. The structural dynamics of silicon \{111\}-type planes were tracked near the vapor/liquid/solid triple-point as the solid/liquid interface fluctuated between roughened, smoothly faceted, and smoothly rounded morphologies. Both the roughened and smoothly faceted configurations were unstable from frame-to-frame while the faceted configuration was observed to persist for as many as three consecutive time-steps. Adjacent to the triple-point, the facets $\{100\},\{112\},\{117\}$, and $\{113\}$ were observed along the interface between with the solid and liquid phases.

In contrast to previous experimental and theoretical work at disordered solid/solid interfaces, no characteristic length between interfacial fluctuations was 
observed. The magnitude of the planar motion of individual $\{111\}$ planes at the interface between a stable $\{113\}$ silicon facet and liquid-phase aluminum alloy showed a bias to quantize changes in lengths corresponding to multiples of the $\{113\}$ inter-planar spacing. This bias towards quantized fluctuation magnitudes has not been shown in previous work modelling the fluctuations present at solid/liquid interfaces in metallic systems; this quantization is likely a result of the strong directional nature of the covalent bonds present in the silicon lattice. In contrast to previous experimental and theoretical work at disordered solid/solid interfaces, no characteristic length between interfacial fluctuations was observed.

Finally, a metastable copper precursor phase was observed to heterogeneously nucleate at the boundary between the solid and liquid phases. These copper islands ranged widely in length along the interface, from $4-132 \AA$; the range of distances that they extended off of the interface into the liquid phase ranged from 2-13 $\AA$. Each copper island observed shared the same $(1 \overline{1} 3)_{S i}\left\|(076)_{C u} ;[110]_{S i}\right\|(100)_{C u}$ orientation relationship with the silicon substrate below. 


\section{Acknowledgements}

First, I must thank my parents for encouraging the innate curiosity of a toddler and helping to develop it into lifelong pursuit. So many people are scared by what they don't know; I hope to always remain excited by it.

To my friends, you all mean the world to me; without your support through this journey I surely would have wandered over a cliff or two. Thank you for the good times, the shared adventures, and the support.

My dearest Emma, you've been a fantastic partner throughout the ups and the downs of our journey together through Virginia. I am so very lucky to have you in my life and I look forward to what our future holds in store for us. I love you.

Finally, I would like to express my deep feelings of gratitude to Professor James Howe. Throughout our time together, you have been a infinite well of knowledge, patience, and encouragement. Thank you for sharing your passion of materials science with me and for giving me this opportunity to work alongside you. 


\section{Contents}

Abstract

Acknowledgements $\quad$ iv

List of Figures vii

List of Tables viii

$\begin{array}{ll}\text { Abbreviations } & \text { ix }\end{array}$

1 Introduction 1

2 Background $\quad 3$

2.1 The Structure of Solid/Liquid Interfaces . . . . . . . . . . . . . . . 3

2.2 Dynamic Behavior at the Interface Between the Solid and Liquid Phases . . . . . . . . . . . . . . . . . 7

2.2.1 Equilibrium Dynamics at the Solid/Liquid Interface . . . . . 7

2.2.2 Non-Equilibrium Dynamics at the Interface Between the Solid and Liquid Phases . . . . . . . . . . . . . . . 9 9

2.3 Chemistry of Solid/Liquid Interfaces . . . . . . . . . . . . . . 13

3 Materials and Methods $\quad \mathbf{1 5}$

3.1 Material Selection and Sample Preparation . . . . . . . . . . . . 15

3.2 in situ TEM Investigation . . . . . . . . . . . . . . . . . . . . 17

3.3 Data Processing Methods . . . . . . . . . . . . . . . . . . 21

4 Experimental Results and Discussion $\quad 23$

4.1 Structural and Chemical Analyses . . . . . . . . . . . . . . . 23

4.1.1 Spatial Distribution of the Atomic Species . . . . . . . . . 24

4.1.2 Microstructure of Melting Phases . . . . . . . . . . . . . 29

4.2 Dynamic Interfacial Behavior Adjacent to the Solid/Liquid/Vapor

Triple-Point . . . . . . . . . . . . . . . . . . . . 32

4.2.1 Experimental Overview . . . . . . . . . . . . . 32 
4.2.2 Frame-by-Frame Analysis . . . . . . . . . . . . . . 33

4.2.3 Interfacial Phenomena at the Triple-Point . . . . . . . . . 37

4.3 Solid/Liquid Interfacial Dynamics Along a Silicon $\{113\}$ Facet . . . 43

4.3.1 Experimental Overview . . . . . . . . . . . . . 43

4.3.2 Data Processing . . . . . . . . . . . . . . . 43

4.4 Heterogeneous Nucleation of Copper on the Silicon $\{113\}$

Solid/Liquid Interface . . . . . . . . . . . . . . . . . . 49

4.4 Experimental Observation . . . . . . . . . . . . 49

4.4.2 Determination of the Structure of the Copper-Silicon

Solid/Solid Interface . . . . . . . . . . . . . 50

4.4.3 High-Resolution TEM Image Simulation . . . . . . . . . . . 52

4.4.4 Characterization of the Copper Nuclei . . . . . . . . . . . 55

5 Summary and Conclusions $\quad \mathbf{6 0}$

5.0.5 Motion of $\{111\}$ Planes at the Triple-Point . . . . . . . . 61

5.0.6 $\{113\}$ Solid/Liquid Interfacial Behavior . . . . . . . . . . 62

5.0.7 Copper Island Observation and Characterization . . . . . . . 62

5.1 Future Work . . . . . . . . . . . . . . . . 63

A MATLAB Algorithm 'measuremement.m' 65

$\begin{array}{ll}\text { Bibliography } & 72\end{array}$ 


\section{List of Figures}

2.1 Schematic of the Solid/Liquid Interface . . . . . . . . . . . . 5

3.1 Aluminum-Silicon binary phase diagram . . . . . . . . . . . . 16

3.2 SEM micrograph of the alloyed nanoparticles . . . . . . . . . . . . . 18

4.1 EFTEM map of elemental distributions within a $250 \mathrm{~nm}$ particle . . 26

4.2 TEM micrograph showing a $\epsilon_{2}$ precipitate on the oxide shell. . . . . 27

4.3 Liquidus projection of the ternary Al-Si-Cu system . . . . . . . . . 29

4.4 HRTEM of interfaces near the vapor/liquid/solid triple-point . . . . 30

4.5 Tableau of frames showing the dynamic behavior near triple-point . 34

4.6 Difference image showing net change at the triple-point . . . . . . . 39

4.7 A highly faceted configuration of the primary silicon particle . . . . 41

4.8 Plot of planar stability near the triple-point . . . . . . . . . . . . . 42

4.9 Solid/liquid interfacial dynamics along silicon $\{113\} \ldots$. . . . . . . . 44

4.10 Heat map of $\{111\}$ planar lengths at the solid/liquid interface . . . 45

4.11 Heat map of frame-to-frame changes in $\{111\}$ planar lengths . . . . 47

4.12 Histogram of changes along the solid/liquid interface . . . . . . . . 48

4.13 Copper nuclei forming at the solid/liquid interface . . . . . . . . . . 51

4.14 Atom-row matching between silicon $\{113\}$ and copper $\{076\}$ facets 52

4.15 High-resolution image simulation of copper islands on silicon . . . . 54

4.16 Histogram of the distribution of copper island heights . . . . . . . . 56

4.17 Histogram of the distribution of copper island widths . . . . . . . . 56

4.18 Plot of percentage of interfacial coverage by $\mathrm{Cu}$ islands . . . . . . . 57 


\section{List of Tables}

3.1 Composition of the AA390 nanoparticles determined by ICP-AES . 17

4.1 Parameters used in the collection of EFTEM images . . . . . . . . 25 


\section{Abbreviations}

$\begin{array}{ll}\text { SEM } & \text { Scanning Electron Microscopy } \\ \text { TEM } & \text { Transmission Electron Microscopy } \\ \text { HRTEM } & \text { High Resolution Transmission Electron Microscopy } \\ \text { EFTEM } & \text { Energy Filtered Transmission Electron Microscopy } \\ \text { EDS } & \text { Energy Dispersive X-ray Spectroscopy } \\ \text { EELS } & \text { Electron Energy Loss Spectrscopy } \\ \text { ICP-AES } & \text { Inductivley Coupled Plasma Atomic Emission Spectroscopy } \\ \text { GIF } & \text { Gatan Image Filter } \\ \text { FFT } & \text { Fast Fourier Transform } \\ \text { MD } & \text { Molecular Dynamics } \\ \text { FCC } & \text { Face Centered Cubic } \\ \text { CCD } & \text { Charge Coupled Device } \\ \text { CBED } & \text { Convergent Beam Electron Diffraction } \\ \text { GPL } & \text { GNU Public License } \\ \text { GIMP } & \text { GNU Image Manipulation Program } \\ \text { MEMS } & \text { MicroElectroMechanical Systems }\end{array}$


It is with a heavy heart that I must dedicate the entirety of this work to a loyal friend who was an eternal wellspring of joy, laughter, and the very best of times. Huckleberry, you were the best friend a man could possibly ask for. With every passing day I miss you and the smile you perpetually brought to my face; rest in peace my friend. 


\section{Chapter 1}

\section{Introduction}

The structure and properties of solid/liquid interfaces are of interest in fields ranging from industrial-scale materials processing to nano-scale drug delivery methods. The structural, chemical, and electrical properties of the interfaces have yet to be fully understood on a fundamental level. The atomic length scales and highly transient nature of such systems has thus far inhibited thorough experimental investigation of them.

The atomic arrangement at the solid/liquid interface transitions from disordered both in spatial and temporal dimensions in the liquid-phase to highly ordered in both dimensions in the solid-phase; this abrupt change in environment occurs over the distance of a few atomic lengths. Knowledge of this transition in properties will aid the understanding of crystal growth/dissolution processes.

To date, our knowledge of the structural details of solid/liquid interfaces comes largely from physical and computational models. Experimental observation of this transition between the two condensed phases of matter has proven difficult and limited results have been reported in the literature ${ }^{1-3}$.

Generally speaking, interfacial phenomena can be classified as either static or dynamic processes; and within each of these classifications, there are equilibrium and non-equilibrium behaviors present. This goal of this work is to increase our understanding of the dynamic behaviors present at equilibrium at the solid/liquid 
interface. To accomplish this, aluminum-silicon alloy (AA390) nanoparticles were used for in situ heating experiments within the transmission electron microscope (TEM). Interfaces between solid primary silicon particles and the liquid-phase alloy were studied at high-resolution and the new interfacial phenomena observed were characterized and are presented in this thesis. 


\section{Chapter 2}

\section{Background}

\subsection{The Structure of Solid/Liquid Interfaces}

At the interface between a crystalline solid and liquid composed of the same atomic species, the differences in condensed phases on either side are significant. The crystalline lattice of the solid phase has well defined long-range order, as well as rotational and translation symmetries. The position of each atom within the lattice is relatively fixed in space. For a given lattice, the number of bonds per atom is highly regular throughout the entire crystalline volume.

On the liquid side of the interface, there is a distinct lack of rotational and translational symmetry beyond even a first nearest-neighbor. The number of bonds per atom varies from 5 to $11^{4}$; this number not only varies from atom to atom, but also within relatively short intervals of time. The prevalence of long-range order in the solid phase is in direct contrast to the dominance of short-range order in the liquid phase. The dense packing of small clusters of atoms is the dominant structural organization. A variety of cluster shapes and sizes occur with the most common being four atoms arranged in a tetrahedron. Characterization of the structure of liquids is most commonly described in reciprocal space and carried out with diffraction based methods ${ }^{5}$. The diffraction patterns obtained 
from liquids show radial symmetry with strong decay between the first, second, and third nearest-neighbors ${ }^{6}$.

The boundary between the solid and the liquid states of matter must connect the structural and thermodynamic differences across the interface. Numerous models have been constructed to study the structural details of solid/liquid interfaces. The first model of the structural change at the interface was constructed by Turnbull, who assembled a random packing of hard spheres representing liquid atoms onto a close-packed array of hard spheres representing the crystalline solid and then examining the structures that formed at the interface ${ }^{7}$. Spaepen modified this approach to maximize the short-range density/order within the liquid phase, and this resulted in the first observation of layering of the densities of liquid-phase atoms parallel to the interface ${ }^{8}$, which exponentially decayed with increasing distance from the crystalline solid. This method and result was repeated computationally by Bonnisent and Mutaftschiev ${ }^{9}$ with the additional finding of a density deficit in the first liquid layer at interface relative to the bulk liquid. This density deficit has been attributed to relaxation of the final crystalline solid layer. Further work has been carried using computational methods; both Monte Carlo (MC) and Molecular Dynamics (MD) methods have shown layering in the liquid directly adjacent to 100 and 111 interfaces in various FCC metals ${ }^{10-12}$.

The first direct experimental evidence of ordering within the liquid-phase was demonstrated by Howe ${ }^{2}$. In this work, the interface between crystalline $\mathrm{Pd}_{3} \mathrm{Si}$ and an amorphous $\mathrm{Pd}_{80} \mathrm{Si}_{20}$ alloy was examined with high-resolution transmission electron microscopy (HRTEM). The micrographs, coupled with HRTEM image simulations, demonstrated that ordering of layers within the amorphous phase extended up to five layers off of the crystalline surface. The intensity profiles taken perpendicular to the interface, correlated to atomic density profiles, closely matched those predicted by Spaepen's model.

The development of in situ heating holders has enabled the study of solid/liquid interfaces at, or near, atomic resolution inside the TEM. The properties of the solid/liquid interface have been examined in a number of materials 


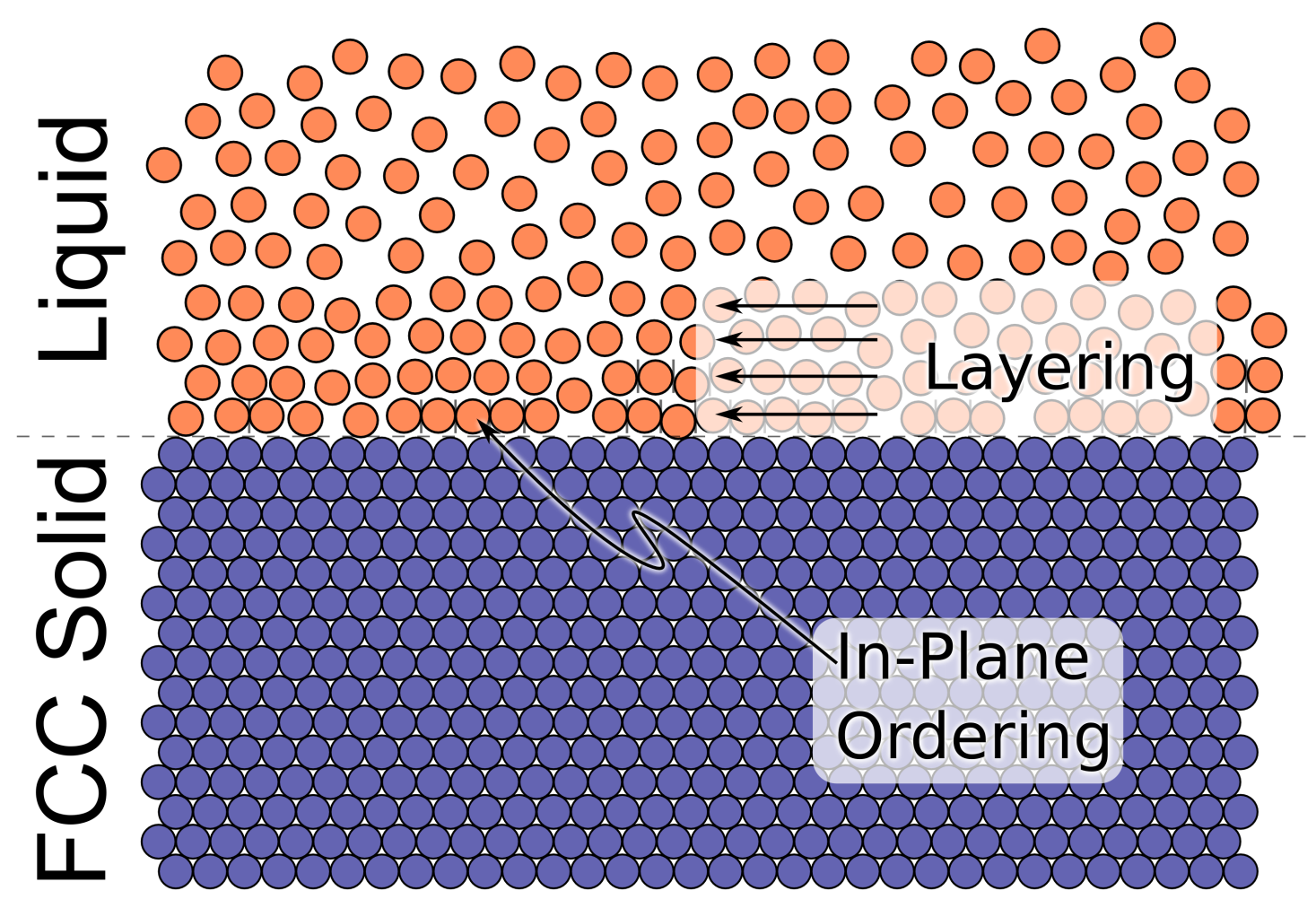

Figure 2.1: A schematic of the solid/liquid interface showing both the layered and in-plane orderings present near the phase boundary.

systems including the interface between a molten metal and a solid semi-metal, aluminum on silicon ${ }^{1,13,14}$; between a molten metal and a solid of another atomic metal species, indium on aluminum ${ }^{15}$; and between and a molten metal and a crystalline ceramic, aluminum on sapphire $\left(\mathrm{Al}_{2} \mathrm{O}_{3}\right)^{3,16}$.

Direct observations using in situ HRTEM of the interface between solid silicon and liquid phase Al-Si alloy were first reported in 1999 by Arai et al ${ }^{1}$. Direct observation of this interface was conducted through the use of solid aluminum and silicon particles, ranging in size from 200-800 nm, placed directly onto the tungsten filament built into a TEM sample rod. Upon heating in situ, the aluminum particles melted and, where in contact with silicon, a liquid Al-Si alloy was formed, thereby creating a solid/liquid interface.

In the Al-Si system, the authors reported that the silicon interface with the liquid commonly formed $\{111\}$ interfacial planes. HRTEM micrographs captured along a (111) faceted solid/liquid showed evidence of ordering in up to three layers of liquid as well as in-plane ordering in the first liquid layer directly adjacent to 
the solid/liquid interface. Additional studies on the same samples demonstrated the ability of the liquid-phase Al-Si alloy melt to facilitate the removal of the amorphous oxide layer natively present on the silicon surfaces in the vicinity of the solid/liquid interface ${ }^{13}$. This phenomenon was repeated and its efficacy demonstrated for both low- and high-index silicon facets including (110), (111), (112), (115), and (773). The authors concluded that this oxide removal was likely the result of the the reduction of the oxide during the formation of an Al-Si complex. Follow-up work with electron energy loss spectroscopy (EELS) indeed revealed a sub-nanometer thick wetting layer of aluminum in the areas where the oxide was removed ${ }^{14}$.

Studies of ordering at the interface between molten aluminum and solid sapphire $\left(\mathrm{Al}_{2} \mathrm{O}_{3}\right)$ have been reported by $\mathrm{Oh}$ et $a l^{3}$ and Gandman et al ${ }^{16}$. In the initial report, ordering was observed at $660{ }^{\circ} \mathrm{C}$ in the liquid aluminum in contact with the $\{0006\}$ facet of $\mathrm{Al}_{2} \mathrm{O}_{3}$. The authors discussed how the effects of phase-contrast delocalization, caused by the spherical aberration of the objective lens, would be convoluted with the image intensity oscillations from the ordering in the liquid. To rule out this possible misinterpretation, HRTEM image simulation work was carried out on a model interface created between the crystalline solid and a liquid generated by MD simulations. With the experimental results carefully analyzed, ordering of six layers of the liquid were reported along the $\{0006\}$ interface.

With the recent adoption of spherical aberration-corrected microscopes, the interface between liquid aluminum and crystalline sapphire was revisited by Gandman et $a l^{16}$. Spherical aberration correction enables one to compensate for and effectively tune-out the detrimental effects of contrast delocalization in phase-contrast images. Without the convolution of contrast oscillations caused by ordering in the liquid and delocalized phase-contrast from the crystalline solid, direct interpretion of images of ordering is possible, allowing smaller amplitude oscillations of density to be correctly interpreted. 
Gandman et al reported on the effects of ordering present within the liquid phase as function of both crystalline facet and temperature ${ }^{16}$. Several HRTEM micrographs of solid/liquid interfaces were presented in which multiple sapphire facets induced differing degrees of ordering within the liquid aluminum. The number of ordered layers parallel to the interface and the degree of in-plane ordering was quantified for $\{0006\},\{1 \overline{2} 10\},\{\overline{1} 014\}$, and $\{10 \overline{1} 2\}$ facets. The number of ordered layers varied from four to seven at $750{ }^{\circ} \mathrm{C}$; by reducing the temperature to $660{ }^{\circ} \mathrm{C}$, an additional eighth layer of ordered liquid was observed parallel to the $\{0006\}$ interface.

\subsection{Dynamic Behavior at the Interface Between the Solid and Liquid Phases}

While the structure of the solid/liquid interface displayed characteristic features, the specific details of its moment-to-moment structure are constantly changing. The atomistic models and experimental results presented in the previous section described the static, or perhaps time-averaged, configurations of the interface. The atomic paths of individual atoms in the liquid phase show significant differences compared to identical atoms located a few nanometers away in the solid phase. The resulting dynamic processes that occur at the solid/liquid interface can largely be divided into equilibrium and non-equilibrium processes, as discussed below.

\subsubsection{Equilibrium Dynamics at the Solid/Liquid Interface}

Subtle processes that occur at an equilibrated interface can provide useful insight into important material properties. For example, from these behaviors, it is possible to determine the entropy, enthalpy, and excess interfacial free energies associated with a particular solid/liquid interface ${ }^{7,8,17}$. Numerous modelling 
methods have been developed to try to calculate such quantities from first principles.

The calculation of interfacial free-energies between an FCC crystalline solid and liquid in a monatomic system has been performed with MD simulations using both a Lennard-Jones potential and a hard sphere potential ${ }^{18,19}$. The interface in these models was formed by the thermodynamic integration method pioneered by Broughton and Gilmer ${ }^{18}$. This computational method determines the excess interfacial free energy by cleaving both liquid and crystalline phases, bringing the cleaved surfaces together, and allowing relaxation to occur all through thermodynamically reversible steps. The excess energy left over from the cleaving process is therefore deduced to be the excess interfacial free energy. Using this method, the interfacial free energies of (100), (110), and (111) interfacial planes for the FCC crystal structure were calculated. The small degrees of anisotropy resulting from these calculations were within the experimental error.

The anisotropy of the crystal/melt interface is an important factor related to microstructural evolution during solidification processes. The small differences between values presented a challenge to the computational community. In 2001, Hoyt et al developed a computational method by focusing on the equilibrium capillary fluctuations at the interface between the solid and liquid phases ${ }^{20}$. Instead of focusing on the interfacial free energy, $\gamma$, this approach calculated local interfacial stiffness. The interfacial stiffness is defined as $\gamma+\gamma^{\prime \prime}$, where $\gamma^{\prime \prime}$ is the second derivative of $\gamma$ with respect to the interfacial normal; this takes into account the changes in $\gamma$ that occur over various radii of curvature, such as those present at the locally fluctuating interface. The focus on the interfacial stiffness is advantageous as it has been shown to be an order of magnitude more anisotropic than $\gamma$. From the simulation results, it is then possible to back-out $\gamma^{20}$.

In these simulations, the interfacial stiffness is determined by observing fluctuations along the interface. The frequencies of the fluctuation modes are quantified and characterized by their wavenumbers. The stiffness for each interfacial configuration is calculated based on the average of all of the fluctuation 
modes observed. Various ordering parameters have been defined throughout studies using this approach ${ }^{20-22}$. The ordering parameters are used to determine the phase to which each and every atom belongs, either the liquid or the solid, and thus to define the interfacial boundary.

The anisotropies of interfacial free energies have been calculated utilizing this method ${ }^{22}$. This analytical method breaks down as fluctuation wavelengths approach the dimensions of the simulation cell. The magnitudes of the fluctuations had a Gaussian distribution and the relaxation times were reported to be on the order of $\sim 100$ picoseconds.

Extending the interfacial fluctuation method, an ab initio-derived potential for aluminum ${ }^{23}$ was used to map out the anisotropy in the interfacial free energy and interfacial stiffness throughout all orientation space ${ }^{21}$. Additional reports have also shown lowering of the solid/liquid interfacial free energy without affecting the anisotropies by alloying between similar metallic species, e.g. copper in nickel ${ }^{24}$, and a lack of solute segregation to the interface within the same alloy system ${ }^{25}$.

At present, virtually all of the reported results in the literature on dynamic behaviors at the solid/liquid interface at equilibrium come from computational models. The only experimental atom-level dynamic behavior reported was on the small fluctuation of a silicon $\{111\}$ plane adjacent to a vapor/liquid/solid triplepoint $^{13}$.

\subsubsection{Non-Equilibrium Dynamics at the Interface Between the Solid and Liquid Phases}

Unlike equilibrium dynamic processes, where there exist several reports in the literature, the possible non-equilibrium dynamic behaviors present at a solid/liquid interface have proven difficult to study. In order to understand some potential behaviors, it is instructive to look at the dynamic behavior present at other types of interfaces that may be similar to solid/liquid interfaces. 
The dynamic process of grain boundary migration was studied using HRTEM at the interface between two grains of pure gold ${ }^{26,27}$. The process of atoms jumping across the interface from one crystalline lattice to another proceeded through a highly localized disordered region, i.e. the grain boundary. Recordings made at 30 frames per second showed that large groups of atoms made the jump across the interface collectively. Frames of no motion across the interface were common, leading the authors to conclude that the large groups of atoms were in fact moving collectively rather than in a piecewise fashion on a timescale faster than the recorded frame rate.

The growth of a crystalline solid into a liquid phase has been compared to the growth of a solid phase into another solid phase across an incoherent boundary. Raffler and Howe reported on massive transformation interfaces in $\mathrm{Ti}-\mathrm{Al}$ across an incoherent boundary ${ }^{28}$. In this transformation process, atoms were observed to jump across the interphase boundary continuously at locations all along the interface. The growth of the transforming phase was continuous, i.e. there was no start/stop growth steps that are frequently observed at coherent and partially coherent boundaries ${ }^{29,30}$. This continuous motion is analogous to growth into a liquid phase; there are no crystallographic constraints on the volume into which the transforming phase is growing. Fluctuations were reported along the interface, some of which spread along the length of the boundary. Two characteristic lengths along the boundary between these fluctuations, 1.15 and $1.61 \mathrm{~nm}$, were reported. By analyzing every tenth frame, the interfacial changes were more pronounced, but the same interfacial characteristics were observed.

Recent advances in direct electron detection-based cameras have enabled frame rates of up to 1,600 frames per second ${ }^{31}$. The motion of atoms near a grain boundary in gold was reanalyzed, making use of the significant increase in temporal resolution afforded ${ }^{32}$. The high temporal resolution was used to demonstrate a number of reversible events that were previously too fast to capture. However, despite the temporal resolution increasing by one and a half orders of magnitude, the fundamental conclusions remained unchanged. These reports of phenomena in multiple experimental systems appearing the same on vastly different time-scales 
indicates fractal relationships through the temporal-dimension; that is to say that the closer that investigators are able to look, the more it appears that the details appear to remain the same.

Dynamic processes associated with the growth of crystalline silicon from the liquid have been observed with HRTEM ${ }^{1,33}$. This growth occurred at the interface between a $\{111\}$ plane and liquid Al-Si alloy. In this growth process, crystalline volumes with lower phase-contrast were observed between the solid and liquid layers; these volumes were interpreted to be in a partially molten state ${ }^{33}$. The growth velocity of the interface was measured to be $20 \mathrm{~nm} \cdot \mathrm{sec}^{-1}$. In order to sustain such a velocity, it was suggested that cooperative motion of atoms in the liquid-phase occurs in order to form the partially molten state directly ahead of the solidifying interface.

A significant advancement in experimental design was realized through the use of aluminum alloy nanoparticles, produced by spray atomization, for in situ TEM experiments. Particles of both hypo and hypereutectic Al-Si alloys have been used to study the solid/liquid interface between the Al-Si liquid and the solid $\alpha$-Al or the solid silicon, respectively ${ }^{34-36}$. These alloys contained copper, magnesium, and other trace elements in addition to the aluminum and silicon. Each nanoparticle was coated in a native amorphous aluminum-oxide layer approximately $3 \mathrm{~nm}$ thick. This oxide layer acted as a containment vessel as the particles were heated beyond the melting temperature of the alloy. The alloy nanoparticles were dispersed onto a standard amorphous carbon TEM grid and placed into a heating holder for observation.

Studies of growth and dissolution of the primary silicon phase within alloy nanoparticles revealed some of the processes at work. Primary silicon particles have been observed to heterogeneously nucleate on the oxide shell containing the liquid phase, while the $\alpha$-Al phase nucleated homogeneously within the molten volume ${ }^{36}$. Consistent with classical nucleation theory, only a small undercooling was required to initiate the heterogeneous nucleation of silicon, while a much larger undercooling $\left(\sim 100{ }^{\circ} \mathrm{C}\right)$ was required to initiate the homogeneous nucleation of 
$\alpha$-Al. Repeated heterogeneous nucleation of silicon on the oxide shell occurred in random locations unless sizeable defect in the oxide shell was present, in which case nucleation preferentially occurred on such a site. A homogeneous nucleation event showed massive growth where virtually the entire volume solidified in two frames; corresponding to a growth rate of $11 \mu \mathrm{m} / \mathrm{sec}$.

Once nucleated, growth of the primary silicon particle was observed in detail. The heating effect of an intense electron beam condensed onto a small particle was shown to be quite useful for fine temperature control without inducing sample drift, as is common when adjusting the temperature of the furnace built into the heating holder ${ }^{37}$. Fine adjustments of the second condenser lens in the TEM, thereby spreading or condensing the electron beam in the sample plane, were used to initiate both growth and dissolution processes observed with a temporal resolution of $1 / 30^{\text {th }}$ of a second. Growth of the primary silicon particle, directed by spreading the beam to reduce the incident radiation intensity, resulted in a strongly faceted particle morphology, indicating strong anisotropy in the interfacial free energies. Meanwhile, dissolution occurred upon condensing the beam; the dissolution process began at the intersection between facets, which dissolved quickly, leaving the particle with a rounded morphology. The growth process of the silicon particle proceeded rapidly on a $\{113\}$ interface and very slowly on a $\{111\}$ interface. Growth of the $\{113\}$ interface occurred virtually instantaneously upon even small changes in beam diameter. Growth of this atomically rough interface continued until it was bound by $\{111\}$ facets. The close-packed $\{111\}$ planes show a much lower sensitivity to small thermal fluctuations.

The structure of the $\{113\}$ interface readily presents many high free-energy sites where solute atoms from the liquid can easily attach and lower the overall system energy; by contrast, the $\{111\}$ interface has the lowest interfacial free energy penalty, thus the driving force for growth is significantly lower and structural rearrangments are more difficult. Growth of the $\{111\}$ facets required the nucleation of a critical size island and subsequent spreading of it along the interface, often observed as step/ledge growth. 
During the study of the growth kinetics of the various facets of the primary silicon particles, rapid interfacial fluctuations were observed on the solid/liquid interface $^{38}$. The magnitude and nature of the oscillations were examined and compared to a wavelike function. The underlying mechanism for the fluctuations, ranging in size from 5 to $35 \mathrm{~nm}$, remains unclear. Additionally, these fluctuations were also observed to occur on the solid/liquid interface between molten and solidified $\alpha$-Al.

\subsection{Chemistry of Solid/Liquid Interfaces}

In addition to the structure and dynamic behavior of solid/liquid interfaces, the chemical nature of the interface is also of significant interest. Due to difficulties in determining chemical compositions at experimental temperatures, very limited information exists on this important topic. Energy Dispersive X-ray Spectroscopy (EDS) is commonly employed in electron microscopy to locally probe the chemical makeup of a volume of material. At elevated temperatures, black-body radiation from the furnace used to heat the sample in situ floods the lithium-drifted silicon of the spectrometer's detector, resulting in a large increase in dark current. This rise in background signal quickly overwhelms the characteristic radiation used for chemical identification and subsequent quantification.

To overcome this physical limitation, Eswaramoorthy et al developed a thermal shield that could be installed onto an in situ heating holder prior to insertion into the TEM column ${ }^{35}$. This shield effectively blocked direct radiative paths between the furnace and the EDS detector, thereby sharply decreasing the thermal noise. Collection of EDS spectra at $550{ }^{\circ} \mathrm{C}$ showed a significant increase in characteristic signal-to-noise ratio from 0.24 without the thermal shield installed to 15.5 with it installed. This 64 -fold increase in signal-to-noise ratio enabled the in situ study of the chemical distributions local to the solid/liquid interface using EDS at experimental temperatures. 
With the development of the thermal shield, the chemical properties across the solid/liquid interface in a hypereutectic Al-Si (AA390) alloy have been probed in situ on the nano-scale ${ }^{35}$. The concentration profiles of aluminum, silicon, copper, and magnesium were determined with a 25-nm-diameter probe across the interface of interest both before and after the melting of the $\alpha$-Al phase. The compositions of aluminum and silicon were found to change in a complementary fashion across the interface in both the solid/solid and the solid/liquid states. The rate of change in the composition, as a function of position, was higher in the solid-solid case; this is expected with the increased diffusivity present at higher temperatures and in the liquid phase. The composition of copper prior to melting was slightly enriched at the interface between the $\alpha$-Al and primary silicon particle, likely due to solute partitioning occurring in the initial solidification process during production of the particles. 


\section{Chapter 3}

\section{Materials and Methods}

\subsection{Material Selection and Sample Preparation}

In order to study the dynamic behavior of the interface between a crystalline solid and a liquid phase, an alloy within the aluminum-silicon system was chosen. By using an alloy with an off-eutectic composition, the interface between the solidified primary particles and the liquid-phase alloy could be observed over a range of temperatures. The Al-Si system is conducive to elemental analysis with both energy dispersive x-ray spectroscopy (EDS) and electron energy loss spectroscopy (EELS). The $\mathrm{K} \alpha_{1}$ characteristic x-ray emission lines for aluminum and silicon are spaced sufficiently far apart to allow quantitative compositional analysis; the $L_{2,3}$ low-loss edges in EELS, however, overlap considerably. The aluminum $L_{2,3}$-edge manifests with a delayed onset beginning at $73 \mathrm{eV}$ and peaks near $100 \mathrm{eV}$; meanwhile, the silicon $L_{2,3}$-edge begins its onset at $99 \mathrm{eV}$ and reaches its maximum at $128 \mathrm{eV}^{39}$. The overlap of these edges limits compositional analysis from the low-loss signal and requires resorting to use of the K-edges located at 1560 and $1839 \mathrm{eV}$ for quantification of aluminum and silicon, respectively. The overlapping $L_{2,3}$-edges, however, were suitable for use of the three-window energy filtered TEM (EFTEM) imaging method in order to spatially map the distribution of these elements within a given nanoparticle. 


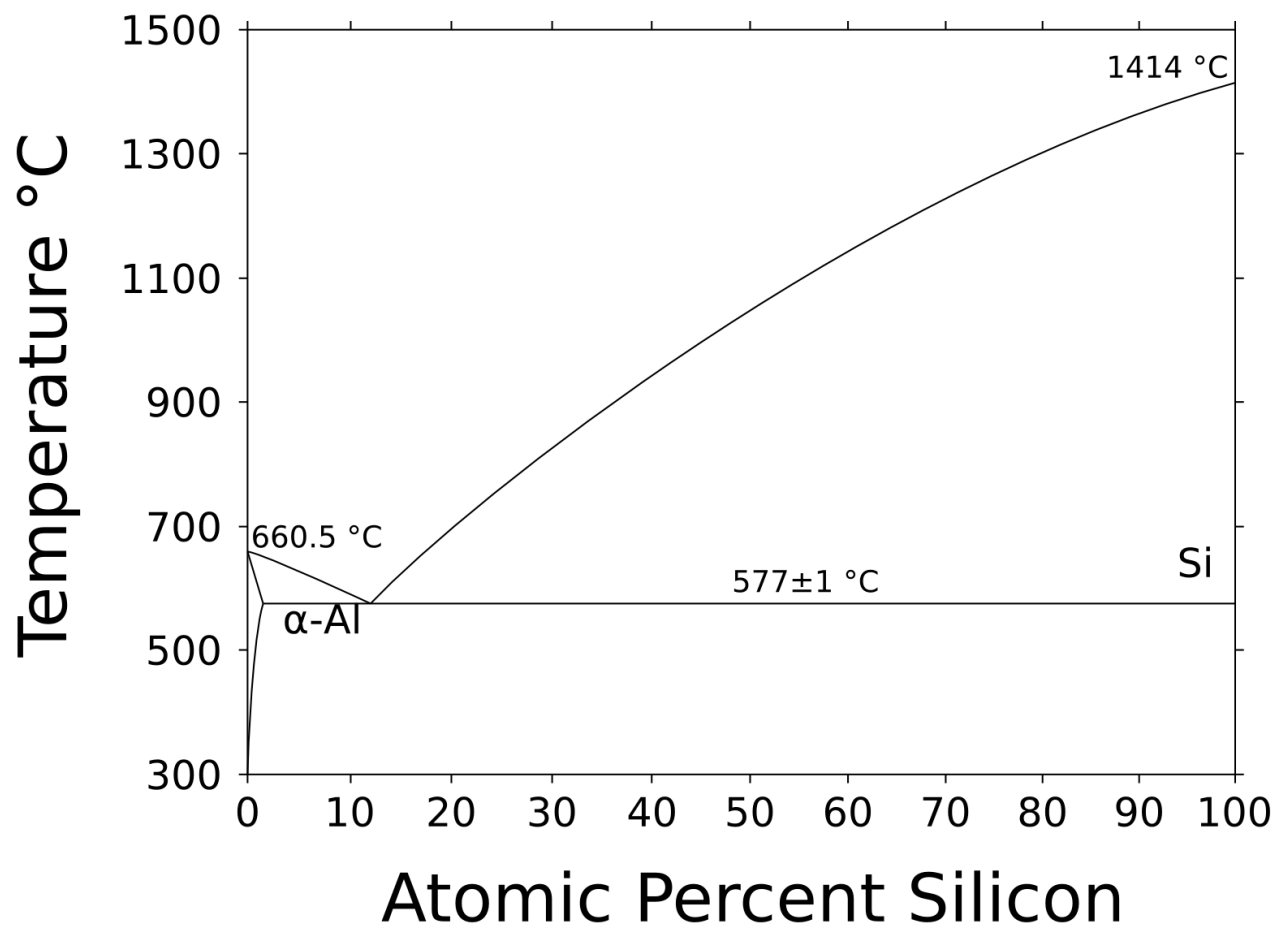

Figure 3.1: The aluminum silicon binary phase diagram ${ }^{40}$

For this series of studies, a hypereutectic aluminum alloy, commercially designated as AA390, with a silicon content of 17.8 atom percent was utilized. The exact composition of the alloy, as determined by inductively-coupled plasma atomic emission spectroscopy (ICP-AES), is listed in Table 3.1. If one considers this as a binary alloy, the Al-Si binary phase diagram in Figure 3.1 shows the alloy forms primary silicon particles beginning at $653{ }^{\circ} \mathrm{C}$ and they continue to grow with silicon solidifying from the liquid phase until $577^{\circ} \mathrm{C}$, when the eutectic composition liquid phase alloy solidifies into both $\alpha$-Al and silicon phases.

To facilitate in situ studies within the microscope's column, the alloy was received in a spray-atomized form from Valimet (Valimet Inc., Stockton, CA) with particle sizes ranging from tens of microns to tens of nanometers. A significant majority of the particles were spherical and were coated by a uniform oxide layer. Some particles were non-spherical and some were observed to have a rough native oxide shell; both of these deviations from the ideal particle morphology are shown in Figure 3.2 below. 
Table 3.1: Composition of the AA390 nanoparticles used throughout this work, as determined by ICP-AES

\begin{tabular}{|c|c|c|c|c|c|c|c|}
\hline Species & Al & $\mathbf{C u}$ & $\mathbf{F e}$ & $\mathbf{M g}$ & $\mathbf{M n}$ & $\mathbf{S i}$ & $\mathbf{T i}, \mathbf{V}, \mathbf{Z n}$ \\
\hline Atom \% & Balance & 1.8 & 0.08 & 0.6 & $<0.05$ & 17.8 & $<0.05$ \\
\hline
\end{tabular}

The native oxide was found to act as a containment vessel for the molten liquid during the in situ studies performed ${ }^{34}$. Particles ranging in size from 100$250 \mathrm{~nm}$ were selected for the in situ experiments described herein; the upper limit on particle size was bound by imaging limitations of HRTEM at $300 \mathrm{kV}$ and the lower limit by the desire to study and characterize behaviors relevant to bulk materials rather than the surface-energy driven phenomena present in smaller nanoparticles.

To prepare samples for in situ TEM observation, $0.5 \mathrm{~g}$ of the spray-atomized alloy was dispersed in $10 \mathrm{~mL}$ of ethanol. The solution was mechanically agitated for 10 seconds, and then ultrasonicated for 24 hours to break up clusters of particles. With this extended ultrasonication time, the smaller particles remained in a suspended state for more than 60 days. To bias the particle size distribution present in a prepared TEM sample towards the smaller particles, the solution was allowed to settle for at least 3 days after ultrasonication was complete. With a pipette, a single drop of the solution was placed on a 400-mesh copper TEM grid coated with a $5 \mathrm{~nm}$ amorphous carbon film and backed by Formvar ${ }^{\circledR}$ (01822, Ted Pella Inc., Redding, CA). The TEM grid was held by anti-capillary tweezers in order to prevent the suspension from being drawn into the tweezers' arms during the solution application and subsequent drying process. The grid was held and allowed to dry for more than 30 minutes before being loaded into the TEM sample holder.

\section{2 in situ TEM Investigation}

A double-tilt heating holder (Model 652.MA, Gatan Inc., Pleasonton, CA) was used to heat samples in situ to $650{ }^{\circ} \mathrm{C}$, just below the liquidus temperature 


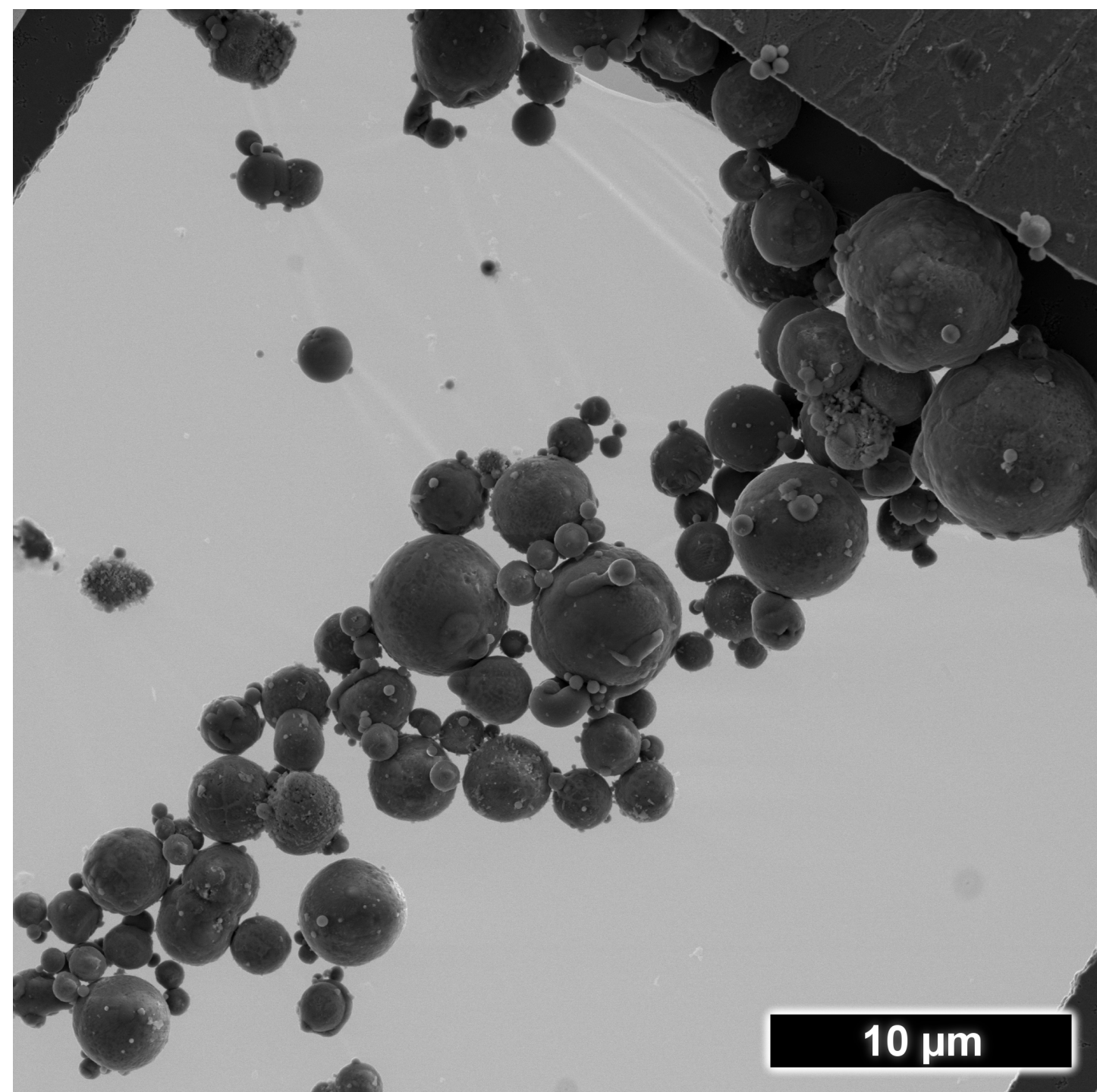

Figure 3.2: A scanning electron micrograph of the alloy nanoparticles distributed on the amorphous carbon film coating a single TEM grid square. The particles show the typical particle size, shape, and oxide morphology distributions.

of the binary alloy system. All samples were held at $650{ }^{\circ} \mathrm{C}$ for at least one hour prior to observation, thereby allowing the system to reach an equilibrated state. This sample holder was equipped with a water cooling system that circulated water throughout the length of the sample rod in order to minimize the thermal expansion of the rod itself. Temperatures were measured with a thermocouple built into the heating holder furnace wall. This thermocouple measures the temperature of the furnace, not necessarily the temperature of a particular region of the sample grid. The actual temperature of a given nanoparticle can not be accurately determined due to a number of complicating factors inherent to in 
situ experimentation. These included temperature variations across the copper grid, distance from a copper grid-bar within a given grid-square, thickness of the amorphous carbon film, and the variable nature of the contact between the carbon film and a given nanoparticle. In addition to these complicating factors, the electron beam of the microscope itself has been shown to impart significant heating depending on the operating conditions of the microscope ${ }^{34}$. This effect has been harnessed and used as a fine temperature control throughout the course of these experiments. Nevertheless, based on practical experience, temperatures within nanoparticles were believed to have been within $\pm 20^{\circ} \mathrm{C}$ of the heating holder's measured furnace temperature.

An FEI Titan 80-300 TEM (FEI Inc., Hillsboro, OR), operated at an accelerating voltage of $300 \mathrm{kV}$ and equipped with an EDAX EDS detector (Model PV9761/85, EDAX Inc., Mahwah, NJ), was used throughout the experimental processes. Nominally, the microscope was operated at spot size 3 in order to provide maximum brightness while minimizing the beam aberrations passed down the column from the electron gun. With the sample held at $650{ }^{\circ} \mathrm{C}$ for an hour before experimentation proceeded, thermal drift was minimized, which aided in the imaging and video recording processes. The local heating control imparted by the incident electron beam did not contribute to stage drift caused by the sample holder arm heating and thermally expanding.

Images were captured on a Gatan Multi-Scan 794 digital camera with a $2 \mathrm{k} \times 2 \mathrm{k}$ charge-coupled device (CCD) mounted at the end of a Tridiem 863P Gatan Image Filter (GIF) electron energy-loss spectrometer. In order to capture video from this device, open-source screen capturing software (CamStudio, GPL license) was installed on the microscope PC. The capture rate was synced with the camera output rate as displayed with the Gatan Digital Micrograph ${ }^{\mathrm{TM}}$ software package. By syncing the capture rate with the display rate, frame duplication was minimized. With the camera operating with the hardware binning set at $2 \times 2$, a $1 \mathrm{k} \times 1 \mathrm{k}$ resolution image was output at a frame rate of approximately 2 frames per second. This temporal resolution is far from ideal for capturing all of the details of the possible phenomena occurring on the interface in these experiments. 
Another drawback of this approach is a loss of dynamic-range in the output signal; while the camera is capable of capturing 14-bit data (i.e. 16,384 gray levels), only 8-bit data (256 gray levels) were recorded due to limitations inherent in modern operating system display drivers. The combination of high spatial resolution and moderate temporal resolution, however, proved more than sufficient to capture a number of previously unreported interfacial phenomena.

Particles of interest were selected once the sample stage was equilibrated at $650{ }^{\circ} \mathrm{C}$ for at least one hour. The particles were initially evaluated based on size, shape, oxide morphology, and the crystallographic orientation of the primary silicon precipitates that had formed at this temperature. In order to observe the largest number of interfacial phenomena simultaneously, silicon particles oriented near $<110>$ zone axes were sought. The tantalum furnace within the in situ heating holder did not have an analytical port milled out of one side-this significantly inhibited the collection of an unobstructed EDS signal. The furnace geometry, coupled with the take-off angle of $20^{\circ}$ between the eucentric-plane of the objective lens and the center of the EDS detector, resulted in occlusion of x-ray emission from a large portion of the TEM grid. In order to increase the area from which a clean signal could be obtained, the sample holder was titled $+15^{\circ}$ along the $\alpha$-axis towards the EDS detector. Even when operating at this optimal geometric condition, work was confined to a small lenticular shaped portion of the sample grid from which an unobstructed EDS signal could be obtained.

Data recording, post-processing, and elemental analysis of the x-ray spectra collected were conducted with the TEM Imaging and Analysis software package from FEI. Spectra were collected for 600 seconds of live-time during which the probe was spread over single-phase regions to minimize knock-on damage that was observed to occur with the use of concentrated probes. All spectra were quantified with the $\mathrm{K}-\alpha$ characteristic lines for $\mathrm{Al}, \mathrm{Cu}$, Si, and $\mathrm{Mg}$. The experimental uncertainties in the computed compositions were less than 0.5 atomic percent.

The EDS signals were used to evaluate the overall particle composition, qualitatively identify phases present, and to locally probe chemistry. The infrared 
radiation from the sample holder, when operated at temperatures above $175^{\circ} \mathrm{C}$, resulted in a large increase in the background noise present in collected spectra. Over $200{ }^{\circ} \mathrm{C}$, characteristic x-ray emission was overcome by the thermal noise. With the temperatures of interest ranging from $500-700{ }^{\circ} \mathrm{C}$, compositional analyses were limited to being conducted either prior to, or after the high-temperature experiments had concluded.

\subsection{Data Processing Methods}

In order to facilitate the analysis of the videos recorded during the in situ experiments, individual frames were extracted using the FFMPEG toolset ${ }^{41}$. The frames from time segments of interest were imported into a stack within the FIJI scientific image-analysis software package ${ }^{42}$. Despite the water cooling of the sample rod and constant furnace temperature, some thermally induced mechanical drift was a constant source of struggle and was present throughout all data-sets collected. In order to deconvolute the effects of thermal drift from actual interfacial growth/dissolution events, the stack of individual frames was aligned using the align slices algorithm included with the Template Matching and Slice Alignment plugin ${ }^{43}$. This algorithm uses a feature present in the entire stack of images as a fixed reference and translates each frame to align this fixed feature. The algorithm makes use of sub-pixel information contained in the red, green, and blue values, which comprise an individual pixel resulting in a higher degree of frame-to-frame stability. In order to correct for the drift in a given sequence of frames, features present within the particle's surface oxide were used; these features were found to be the best stationary reference points as the oxide was not involved in the dynamic processes of interest. The resultant translated stack of images showed the motion of the feature used for alignment to be less \pm 1 pixel over the time periods of interest.

Each frame, once properly aligned, was imported into the GNU Image Manipulation Program (GIMP, GPL Licensed) for analysis. Crystalline samples 
oriented near zone axes allowed interfacial phenomena to be observed at solid/liquid interfaces as well as at solid/liquid/vapor triple-points. The processing of each interface type and observed phenomena required different analysis methods, which are described in further detail in the following chapter. Once the frames were processed appropriately, the transformed data were imported into MATLAB (Release 2013b, Mathworks Inc., Natick, MA). Analyses of these data-sets were conducted using routines written as part of this body of work, see Appendix A. 


\section{Chapter 4}

\section{Experimental Results and}

\section{Discussion}

\subsection{Structural and Chemical Analyses}

Investigation of the spray-atomized nanoparticles in the transmission electron microscope (TEM) yielded a wealth of information from a variety of signals. The orientations of the observed solid phases were analyzed with a combination of electron diffraction and high-resolution imaging. The TEM used throughout this investigation was equipped with a single CCD camera located after the Gatan Image Filter (GIF). This location greatly elongated the camera length when the microscope was used in diffraction mode, rendering electron diffraction patterns unrecordable. However, the electron diffraction patterns remained viewable on the phosphor screen and were used to rapidly assess particle orientation throughout the experimental processes. In lieu of recorded electron diffraction patterns, fast Fourier transforms (FFTs) of images formed by phase-contrast (i.e. high-resolution images) were used for orientation and structural analysis of captured images. 


\subsubsection{Spatial Distribution of the Atomic Species}

Chemical analyses of the spatial distribution of the elements of interest present within the alloy were conducted with both electron energy-loss filtered TEM (EFTEM) imaging and the collection of localized EDS spectra from focused electron probes. Due to the previously discussed limitations, it was not possible to capture EDS spectra at temperatures in excess of $200{ }^{\circ} \mathrm{C}$; as a result, EELS was employed for chemical analyses at experimental temperatures. EDS spectra were collected upon cooling to ambient temperatures and were used for phase identification.

Figure 4.1a shows a bright field TEM image of a $250 \mathrm{~nm}$ diameter nanoparticle at $500{ }^{\circ} \mathrm{C}$. During the heating process, the primary silicon particle present along the bottom of the spherical oxide shell grew as silicon diffused out of the alloyed volumes present. This silicon particle exhibited three facets, emphasized in Figure 4.1c; the darker regions along the right and left-hand sides of the oxide shell were in the liquid state, and the lighter particle contacting the silicon facet at the center was still solid $\alpha-\mathrm{Al}$.

EFTEM elemental maps were recorded to determine the spatial distribution of $\mathrm{Al}, \mathrm{Cu}, \mathrm{Si}$, and $\mathrm{Mg}$ within this particle. To produce these maps, the threewindow method was employed and the images were recorded with the parameters listed in Table 4.1. The window locations specified in the table are the centers of the energy slit relative to the listed onset of the characteristic edge. These maps are not thickness-corrected, and the intensity of the signals are not scaled from elemental-map to elemental-map to compensate for the variable collection times. Within these limitations, however, they provide an accurate visualization of the elemental distributions within and around the nanoparticle.

The elemental maps collected showed the presence of all four of the atomic species within the oxide shell surrounding the particle. Silicon was largely confined to the primary particle and was virtually absent from both the solid $\alpha$-Al and the liquid phase. Copper appeared to be concentrated within the solidified $\alpha$-Al phase 
Table 4.1: A listing of the experimental parameters used within Digital Micrograph for EFTEM elemental mapping

\begin{tabular}{|c|c|c|c|c|c|c|}
\hline Atom & Edge & Onset(eV) & Slit(eV) & Pre1(eV) & Pre2(eV) & Post(eV) \\
\hline $\mathrm{Al}$ & $\mathrm{L}_{2,3}$ & 73 & 10 & -17 & -7 & 5 \\
\hline $\mathrm{Si}$ & $\mathrm{L}_{2,3}$ & 99 & 20 & -32 & -12 & 20 \\
\hline $\mathrm{Cu}$ & $\mathrm{L}_{2,3}$ & 74 & 8 & -14 & -6 & 10 \\
\hline $\mathrm{Mg}$ & $\mathrm{L}_{2,3}$ & 51 & 4 & -7 & -3 & 2 \\
\hline
\end{tabular}

and depleted from the liquid, this is contrary to expectation and the reason why remains unclear. Aluminum was present throughout both the solidified $\alpha$-Al and liquid volumes. A region depleted of aluminum is evident along on the right-hand side of the particle. Viewed in conjunction with the copper map, it is shown that region depleted of aluminum was copper-enriched. Additionally, there was a small layer within the oxide shell that was depleted of aluminum relative to both the outside of the oxide shell and the composition within the particle. The weak signal present within the magnesium map showed segregation along the oxide shell and a tendency to segregate either into or along the surfaces of the primary silicon particle.

The copper signal recorded, while not quantitative, showed significantly stronger signal than expected for the known composition of 1.8 at. \%. Throughout the course of the experimental work, copper was continually over-represented in the elemental analyses performed on samples that were heated in situ. Examination of the TEM grids after extended times (i.e. greater than one hour) at experimental temperatures showed the presence of fine copper particles, on the order of one to three nanometers in diameter, sparsely distributed across the amorphous carbon support film. The source of these copper particles was assumed to be desorption from the TEM grid's solid copper grid-bars, which led to surface diffusion along the amorphous carbon film and ultimately nanoparticle coalescence.

Careful examination of the copper map in Figure 4.1e showed the presence of copper on the amorphous carbon film surrounding the nanoparticle. Adjacent to the alloyed nanoparticle, there was a copper depleted-zone from which the alloy had absorbed all of the available copper. 


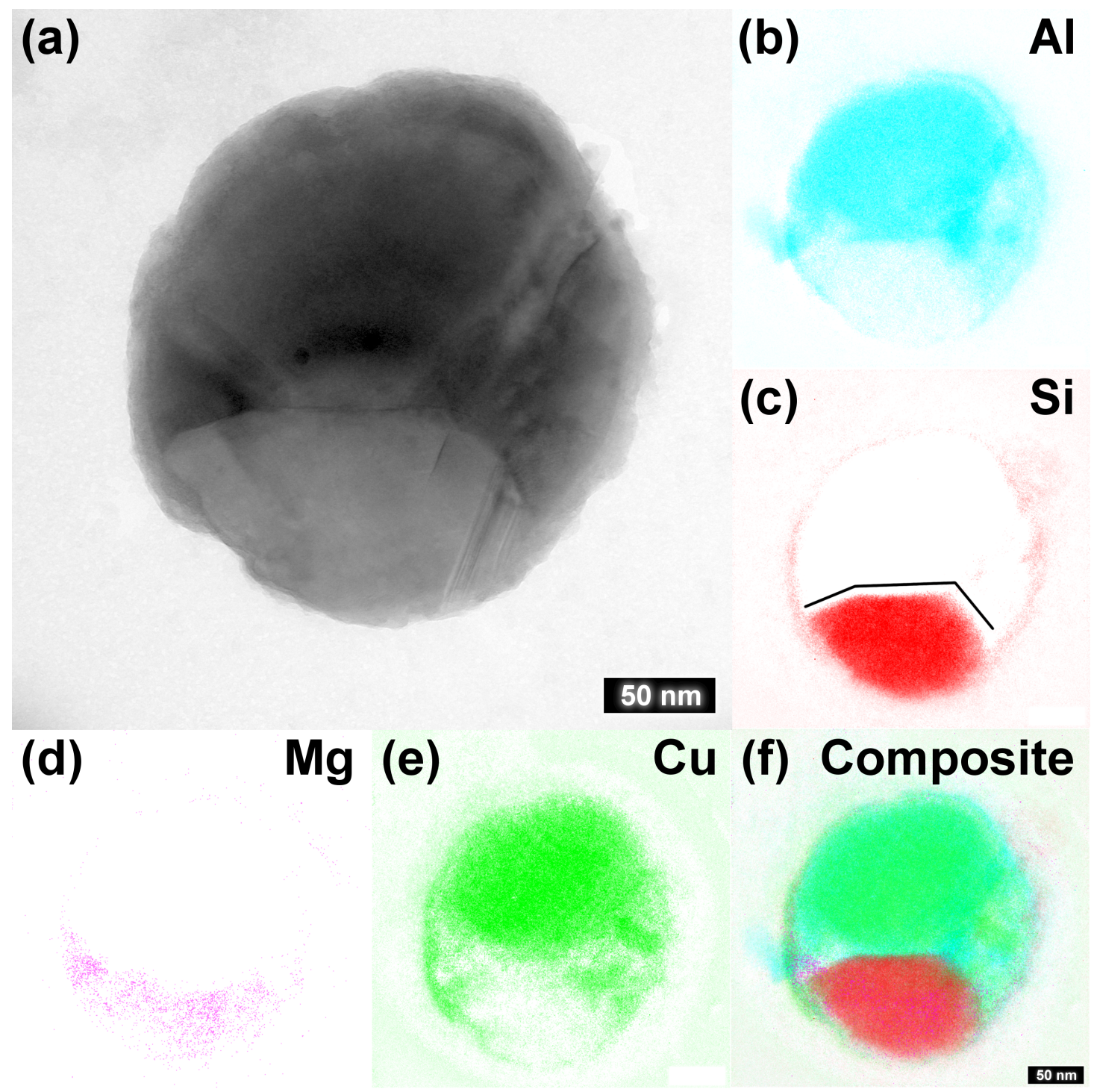

Figure 4.1: (a) Bright field TEM image of a $250 \mathrm{~nm}$ diameter nanoparticle accompanied by EFTEM elemental-maps, (b-f), showing the spatial distribution of the $\mathrm{Al}, \mathrm{Si}, \mathrm{Mg}$ and $\mathrm{Cu}$ elemental signals, respectively; the faceted nature of the primary Si particle is outlined in (c).

The adsorption of copper by the nanoparticle was a continual process, as copper was constantly diffusing from the TEM grid. At times in excess of one to two hours at elevated temperatures, a copper-rich phase was frequently observed to nucleate and grow on the solid silicon and/or the oxide interface with the liquid alloy; a typical precipitate is shown in Figure 4.2.

To identify this unknown phase, and to understand the evolutionary path of the alloy within the nanoparticle, the $\mathrm{Al}-\mathrm{Si}-\mathrm{Cu}$ ternary phase diagram was consulted $^{44}$. The ternary Al-Si-Cu system was used to approximate the behavior 


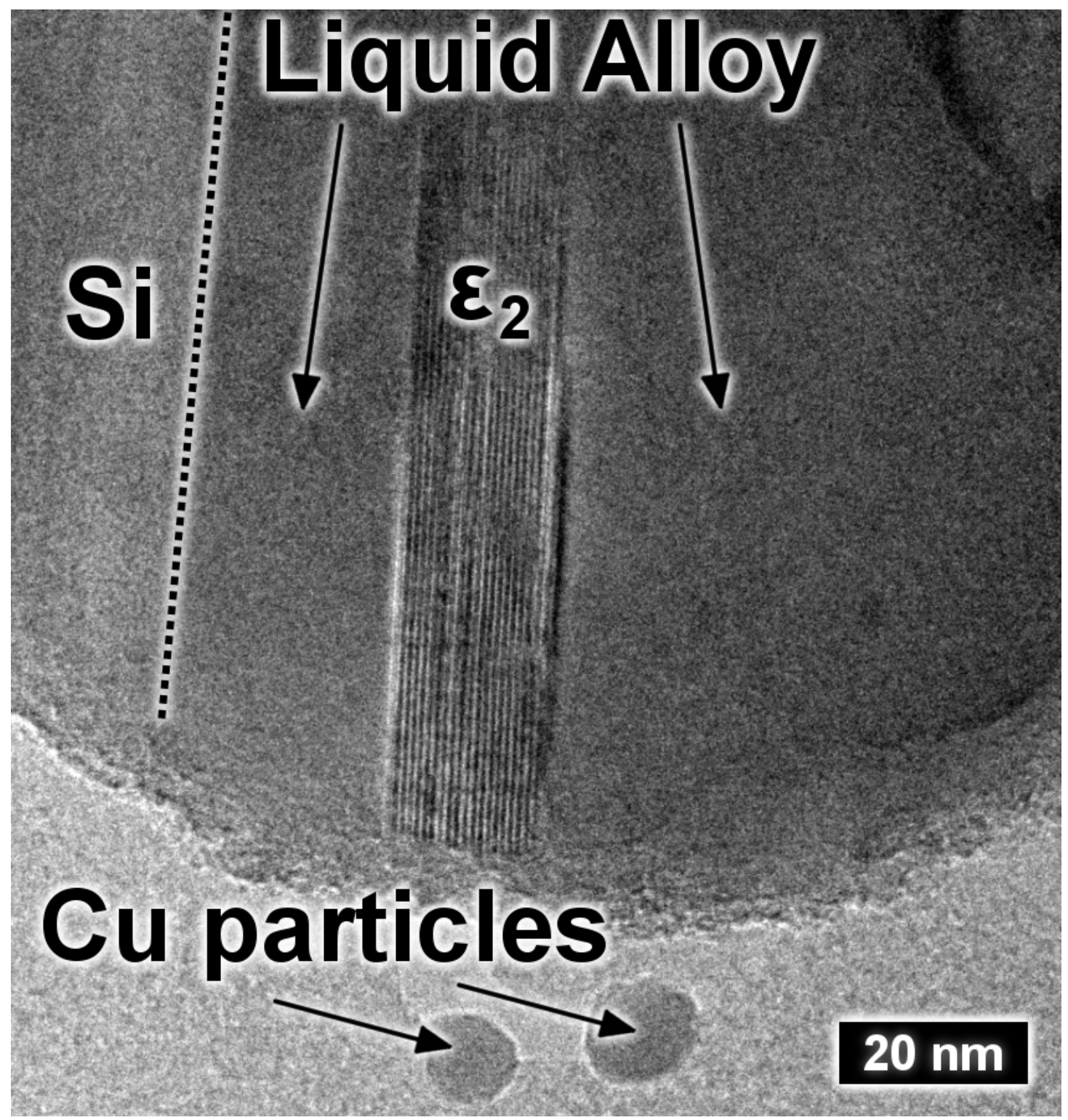

Figure 4.2: A bright field TEM micrograph showing a copper-rich $\epsilon_{2}$ platelet that has nucleated on the oxide shell and grown into the liquid phase parallel to, but not touching, a primary silicon particle visible on the left-hand side.

of the Al-Si-Cu-Mg quaternary system, for which the phase diagram was not available. Figure 4.3 shows the liquid projection within this ternary system with the relevant isotherm, i.e $650{ }^{\circ} \mathrm{C}$, highlighted in red. The initial and final compositions of the liquid measured within the nanoparticle are indicated by the green and purple markers, respectively. As copper diffused into the particle at $650{ }^{\circ} \mathrm{C}$ from the grid-bars, the solubility limit of silicon along the liquidus line decreases from the initial 18 at. $\%$ down to 7 at. $\%$. As the liquid phase reached its copper solubility, the phase boundary on which the purple marker resides in 
Figure 4.3, a solid copper-rich $\epsilon_{2}$ phase heterogeneously nucleated and continued to grow as copper diffused into the system.

Once the $\epsilon_{2}$-phase began to grow, the liquid present within the nanoparticle presumably remained at constant composition. That is to say, as the copper continued to diffuse into the system, the $\epsilon_{2}$-phase grew accordingly and the copper concentration in the liquid directly adjacent to the solid/liquid interface between the solidified silicon and liquid-phase alloy remained constant. The steady-state flux of copper into the system through the oxide shell, through the liquid, and into the growing $\epsilon_{2}$ precipitate likely resulted in the liquid-phase alloy having the same copper concentration over time once the copper sink nucleated. Further, the solubility limit of silicon in the liquid is a function of the copper concentration in the liquid. Observations of the solid silicon/liquid alloy interface showed no net growth/dissolution over extended periods of observation; this lack of growth/dissolution is direct evidence of the stability of the liquid's composition.

The $\epsilon_{2}$-phase $\mathrm{Al}_{2} \mathrm{Cu}_{3}$ intermetallic compound has an ordered hexagonal crystal structure. According to the $\mathrm{Al}-\mathrm{Cu}$ binary phase diagram, this phase only forms at temperatures above $560{ }^{\circ} \mathrm{C}$. The lattice spacing of these precipitates, as measured from HRTEM micrographs, was $2.7 \AA$, which reasonably matches the reported $\epsilon_{2}$-phase $\{0002\}$ interplanar spacing of $2.5 \AA^{44}$. The growth of these precipitates was observed to occur via a ledge mechanism; a new basal plane would nucleate on the long-edged facet at the solid/liquid interface, and would then spread along the face of the precipitate.

The nucleation and growth of copper-rich phases has been observed by previous investigators in this alloy system ${ }^{36,45}$. The previous observations occurred between 565 and $470{ }^{\circ} \mathrm{C}$ and in a system with 1.8 at. \% copper. The intermetallic compound observed previously was determined to be the $\mathrm{Al}_{2} \mathrm{Cu} \theta$-phase by $\mathrm{XRD}$, which, according to the binary $\mathrm{Al}-\mathrm{Cu}$ phase diagram, is stable up to $590{ }^{\circ} \mathrm{C}$.

The significant diffusion of copper into the alloyed nanoparticles observed throughout this investigation has not been previously reported. With further work, this new phenomenon could provide insight into the effects of alloying along 


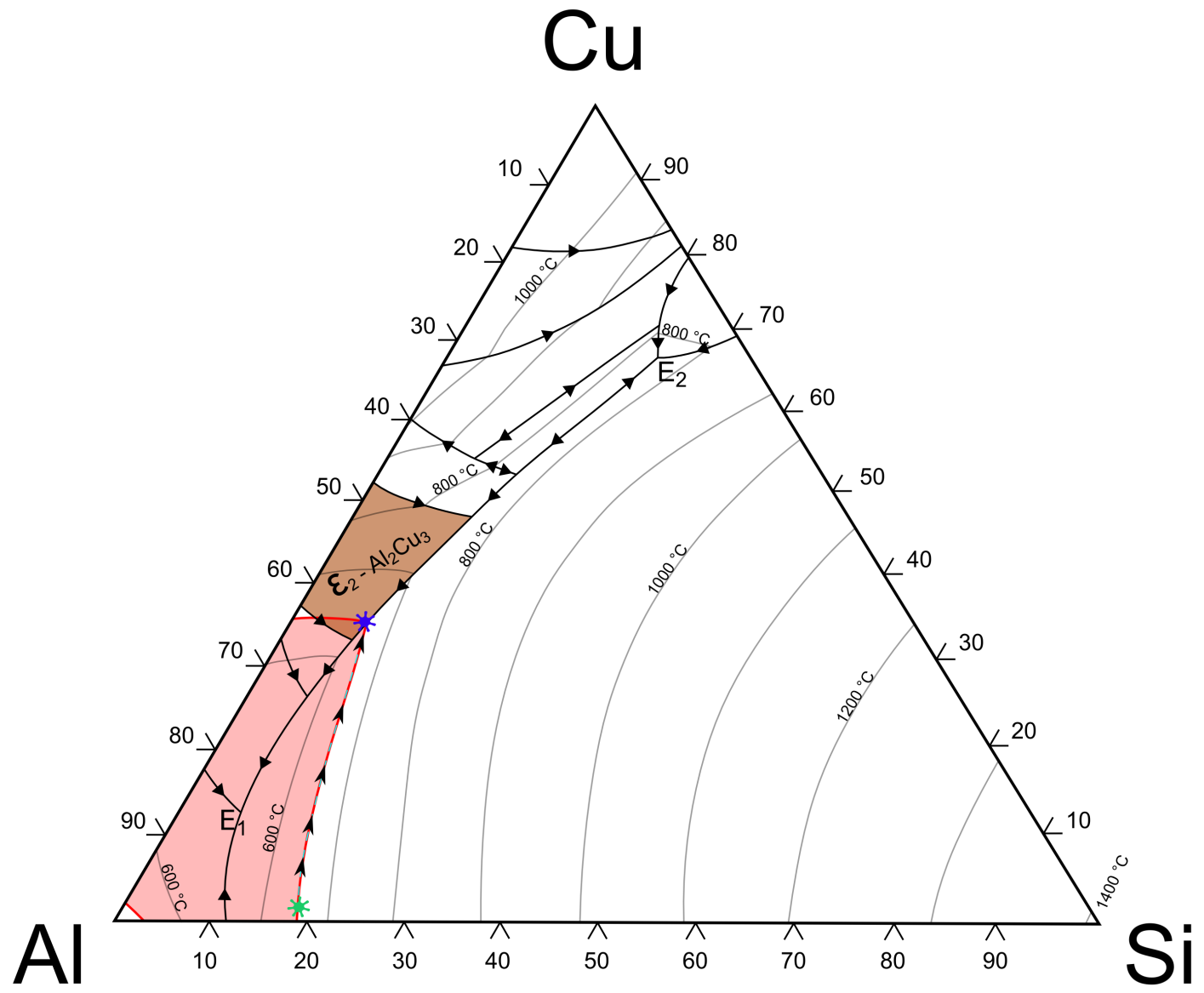

Figure 4.3: The projection of the liquidus surfaces present within the Al-Si-Cu ternary system. The red highlighted region shows the phase boundaries present at the experimental temperature of $650{ }^{\circ} \mathrm{C}$.

a continuous path. Coupled with EFTEM mapping and/or EDS mapping, the evolution of the distribution of the atomic species could be recorded to develop a time-resolved picture of the process of solute redistribution.

\subsubsection{Microstructure of Melting Phases}

Upon heating past the eutectic temperature in the Al-Si binary system, the solid-phase alloy began to melt. According to the binary Al-Si phase diagram ${ }^{40}$, Figure 3.1, the melting process should begin at $577^{\circ} \mathrm{C}$ and be completed at $668^{\circ} \mathrm{C}$. The exact experimental range of temperatures over which this quaternary alloy melted were not precisely quantifiable due to the previously described complications of measuring temperature in a heating holder. The observed phase 




Figure 4.4: A high-resolution TEM image showing the interfaces between the solid silicon, liquid aluminum alloy, and vacuum viewed along the silicon $<110>$ direction.

transformation temperatures of this quaternary alloy matched the binary system to within the $\pm 20{ }^{\circ} \mathrm{C}$ experimental error.

Complete melting of the alloy in the nanoparticles resulted in a solid, faceted silicon particle on the inner oxide shell wall in direct contact with the liquid-phase aluminum alloy. It was this solid/liquid interface, shown in Figure 4.4, that was used throughout the interfacial studies discussed in the following sections.

The crystallographic orientation of the primary silicon particles varied through all of orientation space. Significant effort was put into seeking out 
silicon particles oriented near a high-index zone-axis. Convergent beam electron diffraction (CBED) was used to rapidly screen the silicon particles in appropriately sized nanoparticles. Based on the tendency to facet along the $\{113\}$ and $\{111\}$ planes ${ }^{36}$, it was preferable to view particles along a $<110>$ direction.

The exact orientation of a given silicon particle shifted slightly throughout the period of experimental observation. These shifts were caused by a combination of silicon particle motion on the oxide shell as the shell deformed and degraded under the beam, small reorientations of the whole nanopartcile with respect to the TEM grid, and deformation of the copper TEM support grid itself caused by thermal expansion.

With a silicon particle viewed along a $<110>$ zone-axis, the structural details of the solid/liquid interfaces of interest are readily apparent. Figure 4.4 shows a HRTEM image of a primary silicon particle oriented on the [110] zoneaxis and the interfaces between the solid silicon, liquid aluminum alloy, and vapor phases. Along the left side of the image, the original nanoparticle's oxide structure, on which the silicon particle grew, is visible. The oxide that covered the liquid alloy, as well as the amorphous carbon support film beneath the sample, have been obliterated by the high intensity of the $300 \mathrm{kV}$ electron beam after an extended period of observation. This fortuitous destruction enabled the unhindered observation of the structural dynamics at the triple-point junction of the solid, liquid, and vapor phases. Additionally, at the bottom right of the image, just behind the scale bar, the previously discussed copper rich phase has nucleated and grown into a platelet extending off of the primary silicon particle.

Previous investigations have reported the cleaning of the oxide present along silicon surfaces in the presence of liquid aluminum during in situ experiments ${ }^{13}$. It was stated that the removal of the oxide from silicon surfaces likely proceeded by the reduction of the silicon from the oxidized state to form an Al-Si complex. In previous studies using the same alloy system, the oxide shell was reported to be largely stable at elevated experimental temperatures ${ }^{34}$. The molten metal was observed to be ejected through cracks on occasion, but the complete removal of 
the oxide in any location was not reported at $200 \mathrm{kV}$. In Figure 4.4, imaged at $300 \mathrm{kV}$, the oxide has disappeared from not on only the silicon, but also on the entire surface of the liquid droplet within the field of view. To image this small field of view, the concentration of the electron beam into a small area was required. The increase in accelerating voltage, coupled with the prolonged exposure time and the increase in the electron flux density, would result in an increased amount of beam induced damage relative the work of Storaska and Howe ${ }^{34}$. Based on these facts, it is concluded that oxide removal is likely caused by either a combination of knock-on damage coupled with reduction of the silicon oxide by aluminum, or perhaps solely by the beam knock-on damage itself.

\subsection{Dynamic Interfacial Behavior Adjacent to the Solid/Liquid/Vapor Triple-Point}

\subsubsection{Experimental Overview}

Direct observation of the system at local thermodynamic equilibrium showed incessant dynamic behavior in the area neighboring the solid/liquid/vapor triplepoint (Figure 4.4). In order to establish a condition of thermodynamic equilibrium, the hot stage was heated to $650{ }^{\circ} \mathrm{C}$ and held constant for 90 minutes in order to allow thermal equilibration as well as drift minimization. After the equilibration period, the electron beam was not concentrated on the sample so as to to avoid any appreciable beam-heating effects. Videos, recorded at two frames per second, demonstrated a pattern of behavior present within the system discussed below.

In order to analyze the recordings, sequences of frames were extracted and aligned to sub-pixel precision using the Align Slices plug-in for ImageJ ${ }^{43}$ eliminating the residual drift in the dataset. Once aligned, all frames were rotated to an orientation that aided analysis work. To analyze the behaviors present, the interface between the solid and either the vapor, or the liquid, phase was outlined in each frame using the GIMP software package. 
The dynamic behavior of the crystalline silicon near the triple-point (Figure 4.4) was observed through twenty frames. The silicon in this region transitioned from a stable (111) facet at the solid/vacuum interface through $80^{\circ}$ to a $(1 \overline{1} 3)$ facet, which had stabilized as the preferential solid/liquid interface. Figure 4.5 shows the results of the frame alignment, image rotation, and interfacial boundary determination for the first eighteen frames of this dataset. The orientation of the silicon lattice was approximately 30 milliradians away from a $<110>$ zone-axis, tilted about the $\{111\}$ planes oriented horizontally in the images. The white dots in the image are an unresolved pair of silicon atoms separated laterally by $1.36 \AA$ when viewed along this direction, a so-called silicon dumbbell. Within the field of view, 23 silicon $\{111\}$ planes were visible; every other plane has been indexed on the right-hand side of each frame, starting from the bottom of the image. The atomic structure of the interface was tracked for twenty frames (10 seconds) and the frame-to-frame variations of the planar-steps were analyzed and are discussed below.

The initial condition of the interface, shown in Figure 4.5a, is such that the silicon $\{111\}$ planes are oriented horizontally with the liquid alloy droplet on the left-hand side and the vapor phase along the bottom of the image. Four of the $\{111\}$ planes, labeled 1-4, constitute the solid/vapor interface; while nineteen of the $\{111\}$ planes, those labeled 5-23, make up the solid/liquid interface for the entirety of this series of images. The very bottom plane in the field of view, plane 0 , is observed to have a smaller vertical dimension and is likely a monolayer of an oxide rather than a silicon $\{111\}$ plane. The objective lens was under-focused by approximately $75 \mathrm{~nm}$ throughout this sequence in order to provide strong periodic contrast within the crystalline material.

\subsubsection{Frame-by-Frame Analysis}

During the half-second time-step between Figures 4.5a and 4.5b, the interface was observed to roughen as fifteen of the nineteen $\{111\}$ planes (or step edges) along the solid/liquid interface advanced into the liquid. The advancement of all 


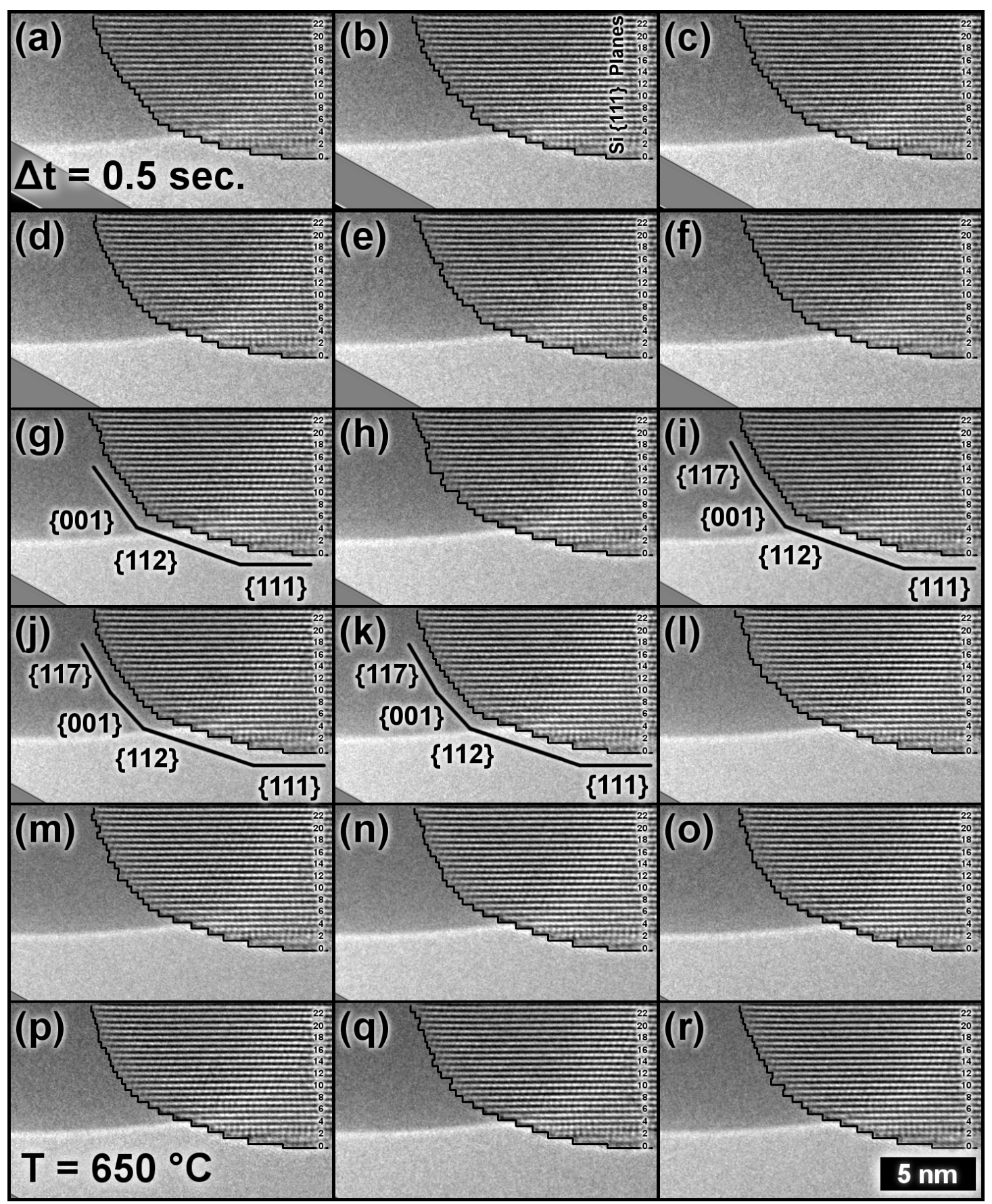

Figure 4.5: A tableau of 18 consecutive frames extracted from a video recording after correction for lateral drift, showing the dynamic behavior of the crystalline solid phase over a nine second interval at the area surrounding the vapor/liquid/solid triple-point. Facets in $(\mathrm{g})$, and $(\mathrm{i}-\mathrm{k})$ have been outlined and labeled 
planes except 5, 8, 19, and 22 created three individual lattice-plane depressions along the interface at the tips of planes 8,12 , and 19. These depressions acted to roughen the interface and increase the total interfacial area (and presumably energy) relative to the smooth structure shown in Figure 4.5a. In the following time-step, Figure 4.5c, all three of the individual lattice planes which were previously depressed in Figure 4.5b were observed to grow into the liquid; this growth was accompanied by the retraction of adjacent $\{111\}$ planes, planes 7,11 , and 18 respectively. This concerted interfacial reshuffling resulted in a smoother, and presumably lower energy, interfacial morphology. In the following frame, Figure $4.5 \mathrm{~d}$, five of the $\{111\}$ planes retracted and the resultant interface was smooth and exhibited a rounded morphology.

From Figures 4.5d and 4.5e, planes 7, 8, and 15 retracted while plane 23 grew into the liquid. The resulting interface had an abrupt two-dumbbell step out into the liquid between planes 8 and 9 along with a small depression created at the end of plane 15. Moving to Figure 4.5f, plane 8 grew out and plane 9 retracted, each by one dumbbell length, while plane 15 grew out to meet the curve of the adjacent interfacial planes. The resulted in a smoother interface with the only notable discontinuity being the two-dumbbell step, which had traversed upwards by one plane to be located between planes 9 and 10 .

Progressing from Figures $4.5 \mathrm{f}$ to $4.5 \mathrm{~g}$, the first evidence of dynamic behavior at the solid/vapor interface was shown as plane 2 retracted by three dumbbell lengths and planes 3 and 4 each retracted by one dumbbell length. The oxide layer, plane 0 , also retracted, while the liquid contact point with the crystal shifted slightly. In previous time-steps, the liquid made contact with the bottom edge of plane 5 while from Figure $4.5 \mathrm{~g}$ onward, the contact point shifted slightly upward onto the tip of plane 5. Further along the solid/liquid interface, planes 7 and 9 grew outward, eliminating the abrupt step that was present in the previous frames; planes 15, 16, and 17 all grew by small amounts as well. The resultant morphology had two crystallographic facets: planes 1 through 7, transitioning through the triple-point, left silicon's (112) plane exposed; and planes 7 through 
17 presented the (001) facet to the liquid phase. Above plane 17, the interface was slightly roughened with no sharp vertices present.

In the next time-step, Figure 4.5h, planes 11, 12, and 14 each grew by twodumbbells and planes 15, 16 and 20 each grew by one. The newly crystallized areas in this frame exhibited weaker contrast than the previously observed changes. The weaker contrast was either from incomplete growth through the thickness, i.e. along the viewing direction, or from a fast event which persisted for only a portion of the half-second exposure time of this particular frame. In either case, the newly crystallized volumes immediately dissolved back into the liquid phase in Figure 4.5i with the exception of growth at the end of plane 20. Careful examination of the solid/liquid interface in Figure 4.5i reveals the presence of three crystallographic facets, the (112) and (001) as seen before, as well as a short segment of a (11̄7) facet, present from planes 14 to 21 .

In Figures $4.5 \mathrm{i}$ through $4.5 \mathrm{k}$, a few small rearrangements occurred along the solid/liquid interface, though the (001), (112), and (11̄7) facets remained intact. The persistence of these facets, despite the dynamic behavior of the system, suggests that the sum of these faceted interfacial planes provides a lower solid/liquid interfacial energy than the less-stable rounded interfacial morphology.

After this 1.5 seconds of relative interfacial stability, a large growth event occurred and is shown in Figure 4.5l, as planes 9 through 18, 20, and 23 extended further into the liquid; the entirety of this newly crystallized volume again exhibited weaker contrast than the adjacent crystalline material. A two-dumbbell length step appeared between planes 14 and 15, and another appeared between planes 22 and 23. As with the previously observed large growth event, shown in Figures $4.5 \mathrm{~g}$ to $4.5 \mathrm{~h}$, the crystalline volume exhibiting weak contrast largely dissolved back into solution in the following time-step. In Figure 4.5m, planes 13 through 23 all retracted by as much as two-dumbbell lengths. The resulting interface, while lacking in large steps, was still relatively rough from plane 11 upward. 
In Frames 4.5m through 4.5p, the interface underwent a very small net growth and a few reshuffling events wherein adjacent planes grew and shrank by equal amounts. The interface in Figure 4.5p is smoothly curved throughout the field of view lacking any notably sized steps. The only interfacial blemish is a small protrusion centered around plane 18, though it is smoothed over neighboring planes rather than being an abrupt discontinuity.

The interface, from Frames 4.5p to 4.5o, showed a net growth only at plane 23. Planes 11, 15, 17, 18, and 20 all receded while planes 12, 14, 16, 20, 22, and 23 all grew. Despite the large number of active planes between these two time-steps, the interface only managed to roughen. In Frame 4.5r, the interface retracted as planes 7 through 10, 12 through 14, 16 through 18, and 20 through 23 all decreased in length; the only plane which increased in length was plane 11 . The result of this concerted dissolution event was a curved interface with a sharp, two-dumbbell length step at plane 11. The subsequent frames, not shown in this figure, resulted in the interface being smoothly curved followed one time-step later by a growth of event of 10 planes with the new growth volume once again exhibiting weaker contrast and a roughening of the interface.

\subsubsection{Interfacial Phenomena at the Triple-Point}

Examining these data, a number of general characterizations are evident. First, of the twenty frames examined, eight of the solid/liquid interfaces were seen to exhibit a smooth morphology and twelve were considered to be rough. The smooth interfacial structures take on one of two characteristic morphologies, either rounded or faceted. The three frames determined to be rounded are Figures 4.5d, $4.5 \mathrm{p}$, and frame 19 (not shown in the tableau); the faceted frames are Figures $4.5 \mathrm{a}, 4.5 \mathrm{~g}, 4.5 \mathrm{i}, 4.5 \mathrm{j}$, and 4.5k. Across the 20 frame dataset, there were no rounded solid/liquid interfaces which persisted from one frame to the next. In contrast, there was a three-frame continuity of a faceted solid/liquid interface present in Figures 4.5i through 4.5k. In addition to the persistent facets in these three frames, the faceting exhibited in Figure 4.5g persisted through a one-frame 
growth-induced roughening event on the interface in Figure $4.5 \mathrm{~h}$, and persisted through to Figure $4.5 \mathrm{k}$.

From these data, it is concluded that the stability of the faceted interfacial morphology is greater than the rounded morphology during the period of observation under the equilibrium conditions present at the triple-point. These stabilities are indicative of differences in the solid/liquid interfacial free energies of the various morphologies. The data described above indicate that the faceted interfacial structure is of lower total energy. The directional, covalent bonding in crystalline silicon leads to anisotropic properties ${ }^{46}$, as reflected in the preferential faceting.

Eswaramoorthy and Howe have previously reported observation of faceted and rounded interfacial morphologies in the same alloy system ${ }^{36}$. In their work, growth and dissolution of the primary silicon particles was directed by spreading/condensing the electron beam. The silicon was observed to rapidly respond to the change in temperature resulting from the change in illumination condition. The growth was reported to proceed rapidly on the silicon $\{113\}$ facet and slowly on the $\{111\}$ facet, after the $\{113\}$ interface grew-out. Dissolution began on the corners between facets and resulted in rounded particles. By oscillating between growth and dissolution these behaviors were repeated several times. The faceted growth and rounded dissolution morphologies were quite pronounced, but on the micrometer-scale.

The rounded and faceted morphologies produced in this work, shown in Figure 4.5, were observed as the structure of the interface fluctuated during equilibrium processes. Small individual growth events produced both rounded, e.g. Figure $4.5 \mathrm{~m}-\mathrm{p}$, and faceted, e.g. Figure $4.5 \mathrm{~h}-\mathrm{k}$ morphologies. The characteristic behaviors reported previously do not appear to apply to the atomiclevel fluctuations about equilibrium at this interface.

In order to examine the net interfacial change over the time period characterized, the first and last smooth solid/liquid interfaces, frames 1 and 19 respectively, were compared. To determine the plane-by-plane changes, a 


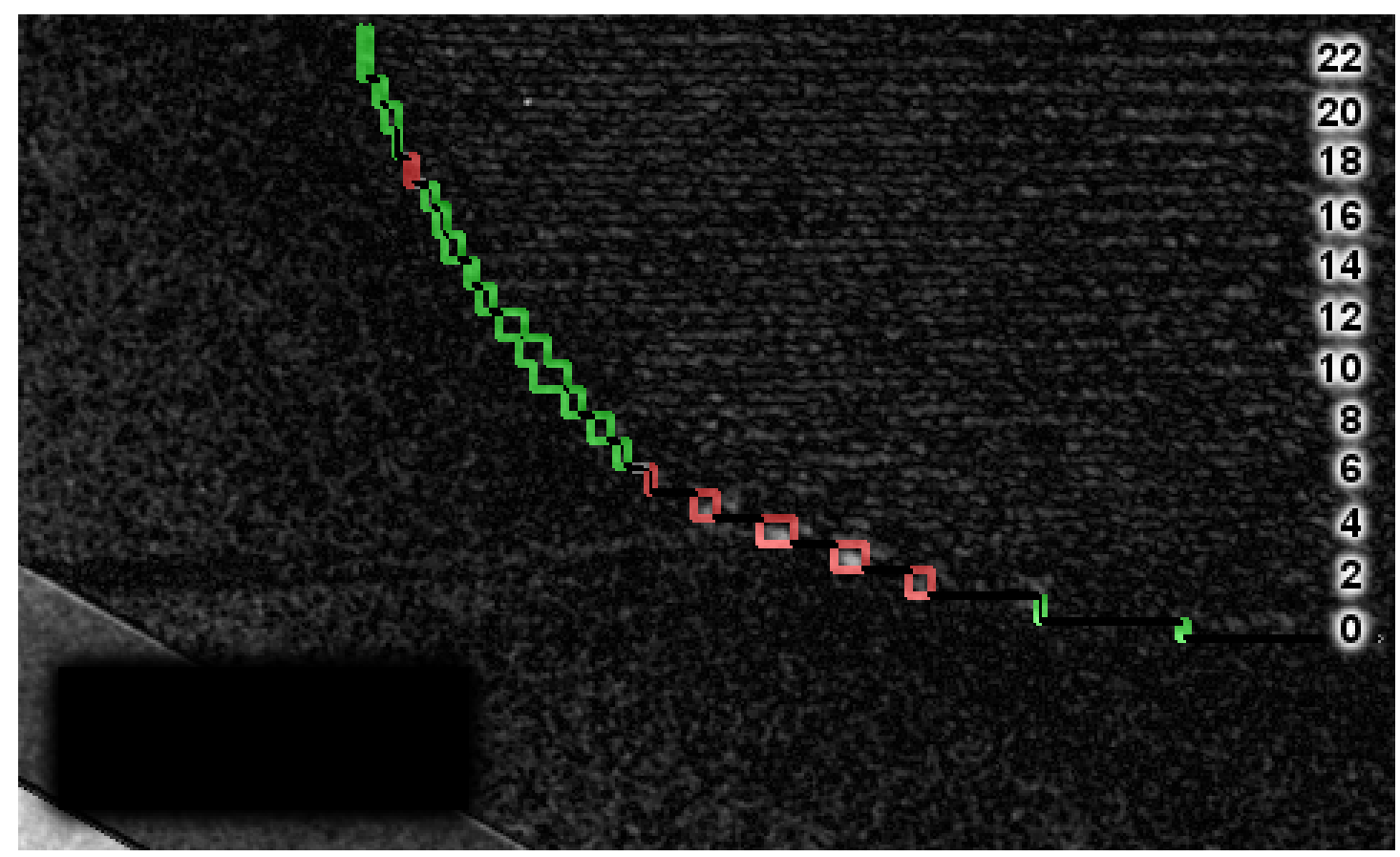

Figure 4.6: A difference image computed from the first and nineteenth frames in the video sequence. The false color overlay shows the net growths in green and dissolutions in red.

difference image was constructed between frames 1, Figure 4.5a, and frame 19. In Figure 4.6, a false-color overlay has been added to ease in the identification of planes that experienced a net growth, outlined in green, and planes which experienced a net contraction, outlined in red. Overall, the solid at the solid/liquid interface was observed to have grown a small amount into the liquid while the solid retracted a small amount along the solid/vapor interface so that the net changes are essentially zero. The net movement observed is well within the frame-to-frame variation in the series of frames shown in Figure 4.5 above.

Throughout the period of experimental observation, three framesFigures 4.5h, 4.5l, and frame 20 (not shown)-had crystalline volumes at the edge of the solid/liquid interface, i.e. at the edge of these steps that exhibited much weaker contrast than the crystalline regions surrounding them. All three of these observed crystalline volumes were produced in newly solidified volumes; none was created through dissolution events. Two possible interpretations of this phenomena are likely. The first possibility is that these frames captured the nucleation of a island extending off of the established solid/liquid interface into the liquid phase. To 
produce the experimentally observed weaker contrast oscillations, the nucleated island would have to extend only partially through the thickness along the viewing direction. The second possibility is that the weaker contrast captured in a single frame was the result of a transient crystallization event which occurred on a considerably faster time scale than the half-second exposure time of each frame. Although either situation can occur, it is possible to distinguish between the two in the present case, as discussed further below.

With the crystalline volume tilted slightly off of a $<110>$ zone-axis, the oscillations in contrast were observed to diminish at the end of a given $\{111\}$ plane in the final nanometer before the solid/liquid interface in all of these frames. This degradation in contrast is consistent with the effect of thickness variation caused by interfacial inclination. Further, the weakly contrasting volume in Figure 4.51 largely dissolved in the following frame Figure $4.5 \mathrm{~m}$. The tip of plane 12 was part of the newly grown volume in Figure 4.51 displaying weaker contrast; examination of Figure $4.5 \mathrm{~m}$ shows that not only did plane 12 persist through this period of time, its display of weaker contrast also remained. Further, the consideration of crystallization through a fraction of the viewing direction at a solid/liquid interface has been examined and simulated in detail ${ }^{33}$. The damped intensity oscillations seen with in Figures $4.5 \mathrm{~h}$ and $4.5 \mathrm{l}$ qualitatively match the behavior of the simulated images reported by Howe and Saka ${ }^{33}$. These factors lead to the conclusion that the crystalline areas exhibiting less intense contrast oscillations are indicative of incomplete crystallization along the viewing direction.

The primary silicon crystal which nucleated and grew on the oxide shell of the original nanoparticle exhibited various crystallographic facets as it transitioned

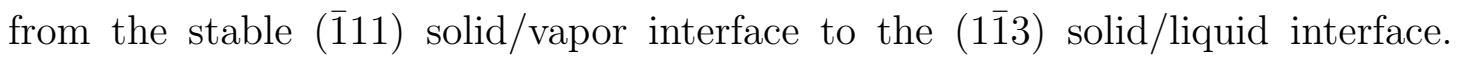
As the solid/vapor interface neared the triple-point, a series of steps formed that constituted a (112) facet. This particular facet persisted throughout the period of observation and was observed to be stable through the triple-point a short distance into the liquid phase. In contrast to the solid/vapor interface, the solid/liquid interface was significantly more dynamic. Along this interface, a range of facets were observed, though most of them were transient in nature. Figure 4.7 


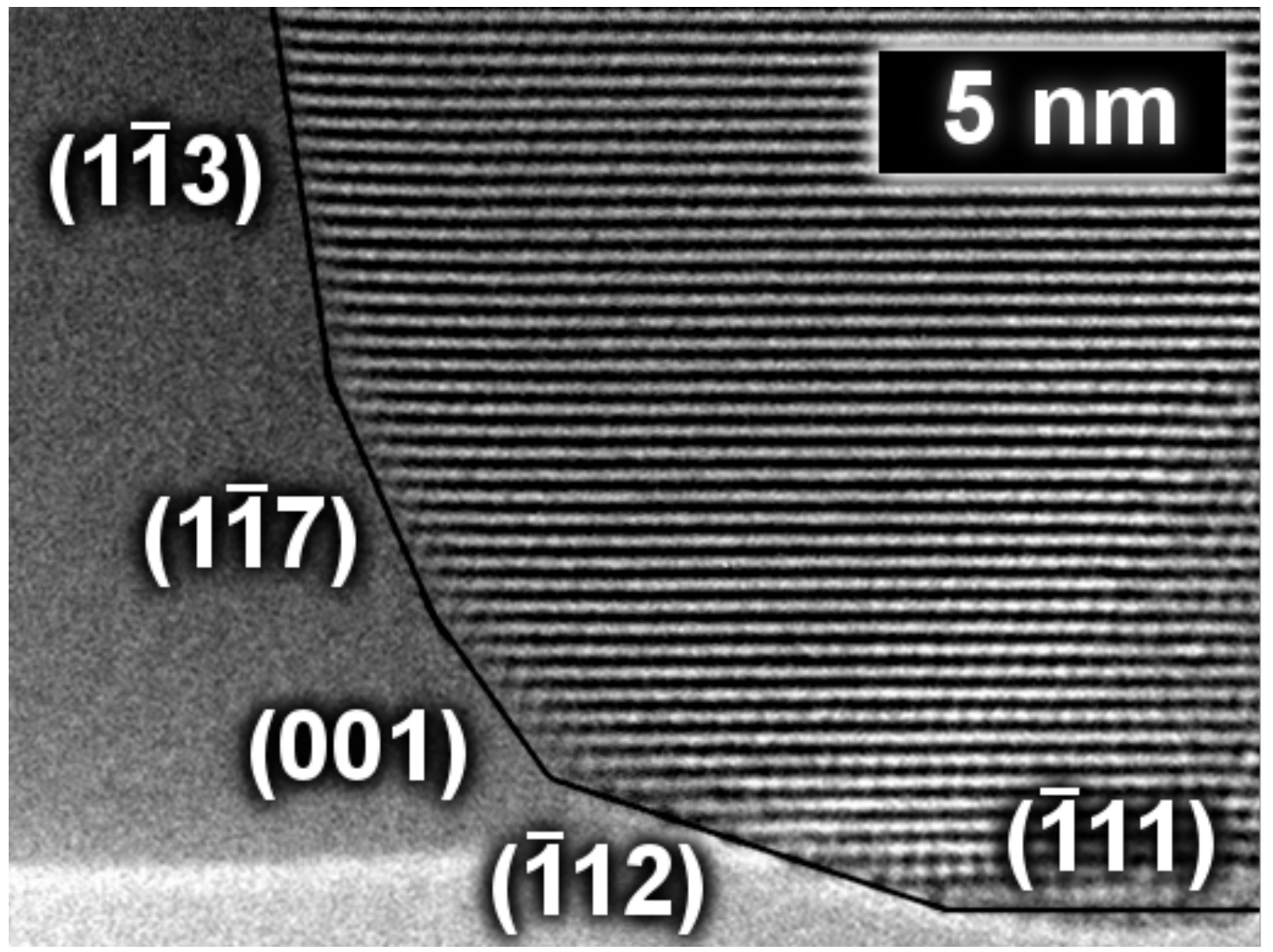

Figure 4.7: An extracted frame showing a highly faceted configuration of the primary silicon particle at the vapor/liquid/solid triple-point

shows an extracted frame in which the interface was captured in a highly faceted

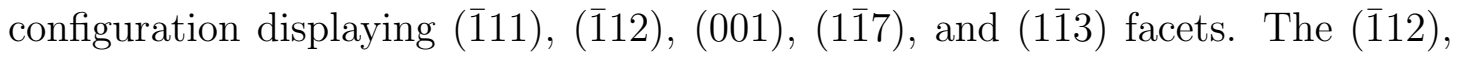
(001), and (1 $\overline{1} 7)$ interfaces between the solid and the liquid were variable, whereas the (113) interface, as shown in Figures 4.4 and 4.7, was persistent throughout the entire period of experimental observation.

Finally, to quantify the activity level of each $\{111\}$ plane for all 23 steps present in Figure 4.5, the frame-to-frame variations were characterized as either positive, negative, or zero net change. In order to account for errors of judgement in determining the exact interfacial plane/steps position's, a non-zero change was defined as the interface moving by three or more pixels. This dataset did not quantify the magnitude of each change, only the character of the change.

The number of frame-to-frame differences resulting in no change versus plane number was plotted, as shown in Figure 4.8 below. Planes 0 through 4 constituted the solid/vapor interface and planes 5 through 23 made up the 


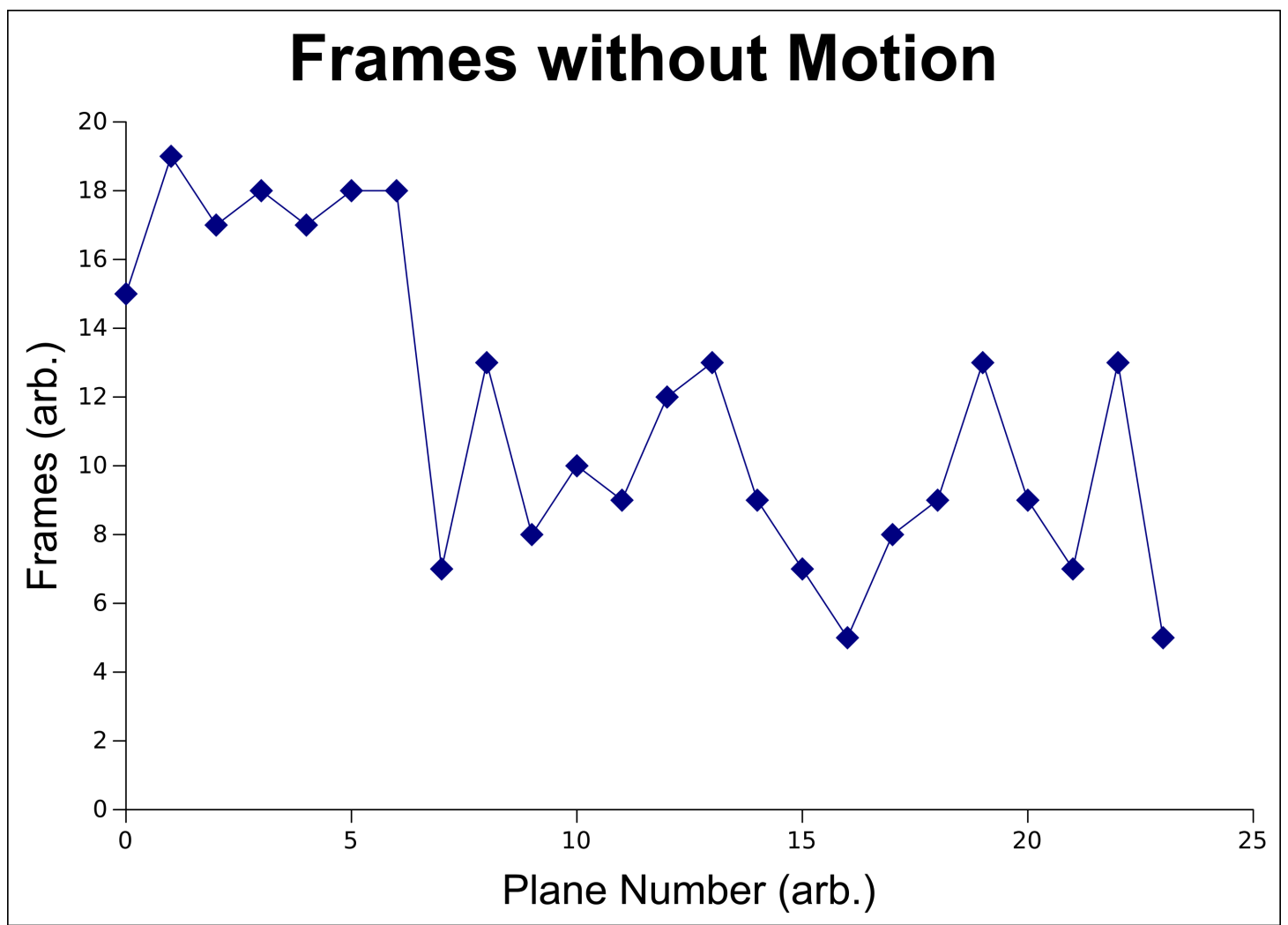

Figure 4.8: A plot showing the relative stability of each plane in the area adjacent to the triple-point.

solid/liquid interface. From this plot, it is evident that the solid/vapor interface was significantly less active than the solid/liquid interface. The transport of silicon atoms to and/or from the solid/vapor interface was significantly reduced relative to the solid/liquid interface. The region of planar stability extended inwards two planes from the triple-point into the liquid phase covering planes 5 and 6 . The stability is attributed to the presence of the (112) facet which was persistent at the vapor/liquid/solid triple-point. While the reason the (11̄2) was stable is not known, it may relate to equilibrium among the various interfacial energies/forces pulling at the triple-point in this location. 


\subsection{Solid/Liquid Interfacial Dynamics Along a Silicon $\{113\}$ Facet}

\subsubsection{Experimental Overview}

Observation of the solid/liquid interface at local thermodynamic equilibrium showed continuous, dynamic behavior of the $\{111\}$ planes terminating along the (11̄3) interfacial boundary between the crystalline silicon and liquid alloy phases, shown in Figure 4.4. In order to establish a condition of thermodynamic equilibrium, the hot stage was heated to $650{ }^{\circ} \mathrm{C}$ and held at this temperature for more than 90 minutes. Additionally, the electron beam was not concentrated on the sample throughout the course of this experiment in order to avoid any appreciable heating from the electron beam.

Videos were recorded at two frames per second at $1 \mathrm{k} \times 1 \mathrm{k}$ imaging resolution. To analyze the frame-to-frame variations, 61 frames were extracted from the video file, imported into GIMP, aligned, and rotated to orient the $\{113\}$ solid/liquid interfacial plane into the horizontal position; in this orientation, the silicon $\{111\}$ planes are oriented approximately $9^{\circ}$ from the vertical. Throughout the period of observation, the interfacial facet remained along the silicon $\{113\}$ plane, and the overall solid/liquid interfacial position remained unchanged, i.e. there was no net growth or dissolution.

\subsubsection{Data Processing}

For all frames, a single pixel mark was placed at the terminus of all 49 of the silicon $\{111\}$ planes within the field of view. The $y$-value of this pixel location was used to generate a schematic of the interface consisting of vertical bars representing the $\{111\}$ planar lengths. In Figure 4.9 frames 57 through 60 are shown along with the schematic generated from the planar termination measurement overlaid. 


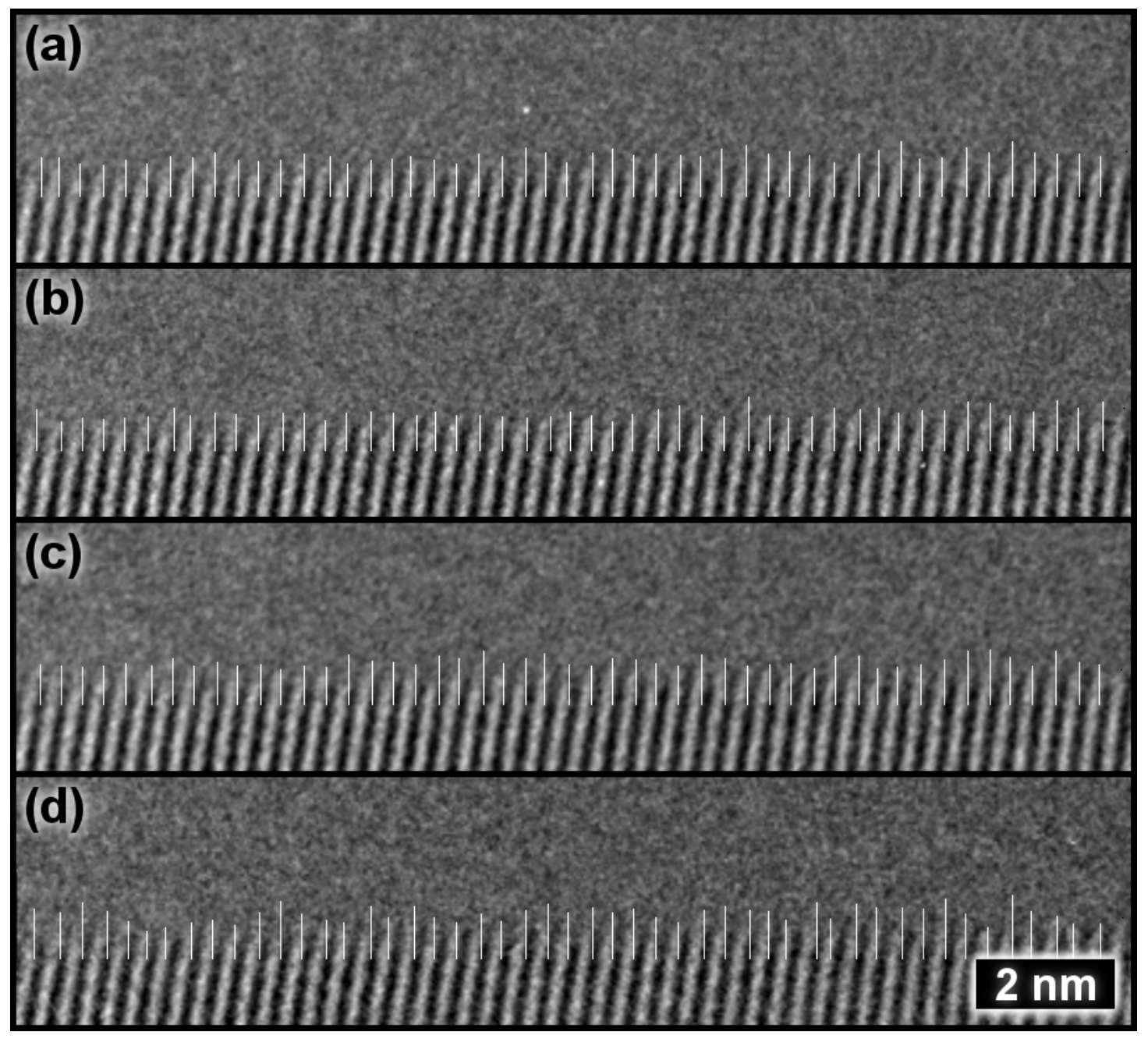

Figure 4.9: Four consecutive frames of the solid/liquid interface showing the dynamic behavior with the planar termination analysis schematic overlaid.

A bespoke software routine, measurement.m (included as Appendix A), was developed and implemented to import the schematics generated in GIMP into a three-dimensional data array in MATLAB. The length of each plane in each frame of the video was organized into a three-dimensional array; the three dimensions being plane number (along the x-direction in the video frames), plane length (the distance along the $\mathrm{y}$-direction in the video frames), and frame number (i.e. time). A heat map was generated to represent this three dimensional dataset, Figure 4.10 below. In this figure, each of the silicon $\{111\}$ planes terminating along the $\{113\}$ facet are represented along the x-axis; the individual time-steps along the $\mathrm{y}$-axis; and the planar lengths, relative to the average interfacial position, are represented in color. 


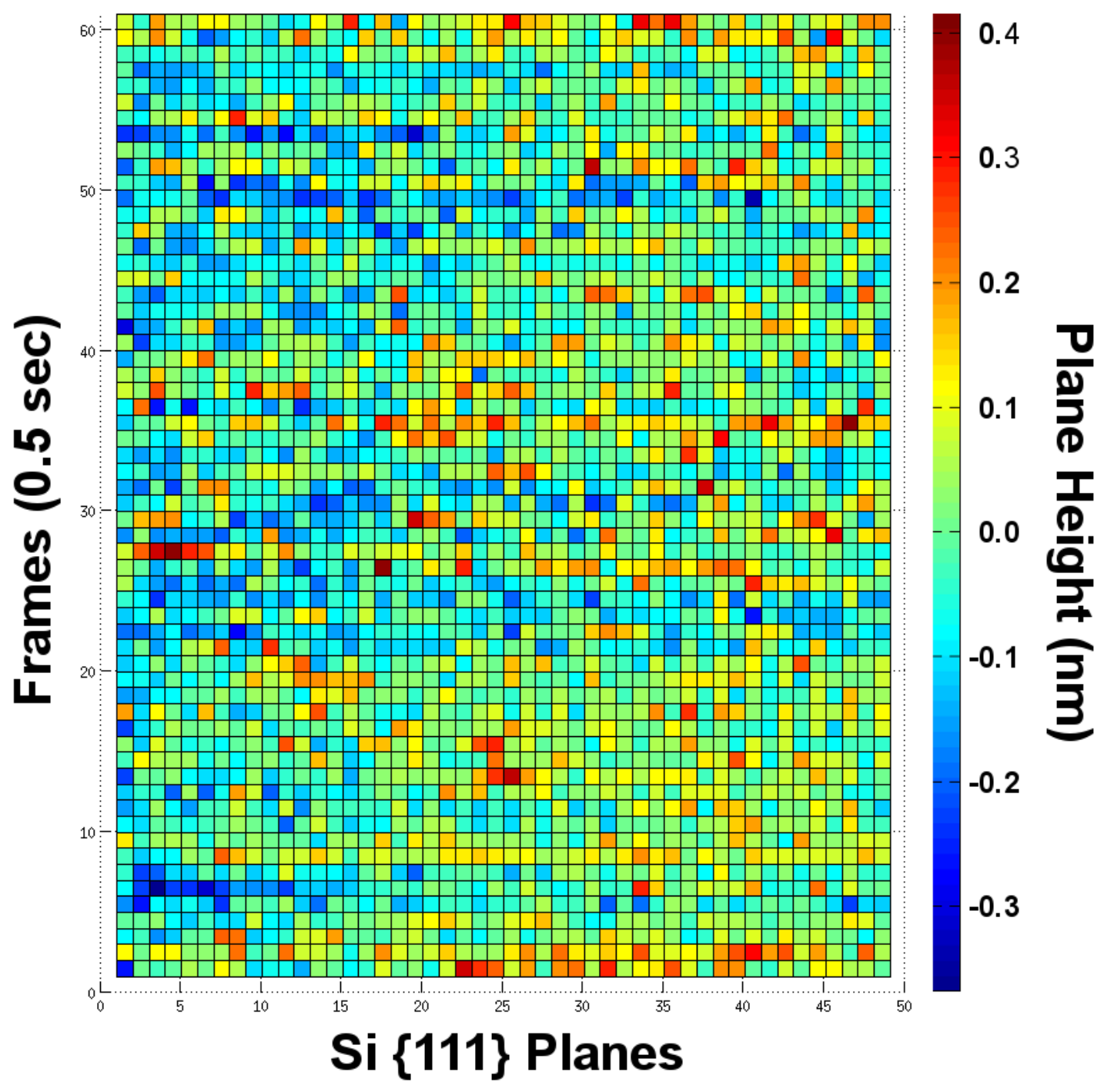

Figure 4.10: A heat map representing the planar lengths of the individual silicon $\{111\}$ along the interface over a period of 30 seconds.

Throughout the time period analyzed, the interfacial motion remains confined to a range of $8 \AA$. This motion occurred equally in both the positive direction, extending into the liquid, and the negative direction, depressing into the solid. Instances of individual $\{111\}$ planes, pairs of planes, and groups of up to five adjacent $\{111\}$ planes were observed to move in both the positive and negative directions.

Previous experimental work on incoherent boundaries between solid phases have shown characteristic spacings between interfacial growth and/or dissolution events $^{28}$. A fundamental wavelength of 1.15 and an additional characteristic 
wavelength $\sqrt{2}$ times the fundamental wavelength, $1.61 \mathrm{~nm}$, were reported. The authors found no crystallographic connection between either the fundamental nor the characteristic wavelength and suggested that further examination may provide additional insight into the origin of this relationship.

In order to determine if fundamental and/or characteristic wavelengths were present between the oscillations at the interface between the crystalline silicon and liquid alloy, one-dimensional fast Fourier transforms (FFTs) were computed along the $\{113\}$ interface for each frame analyzed. These FFTs showed no recurrent characteristic spacing for either the growth or dissolution events observed along the solid/liquid interface. The lack of this behavior at the solid/liquid interface suggests that the characteristics previously observed were partially tied to the crystallographic and/or kinetic specifics of the incoherent solid/solid interface.

To explore the frame-to-frame changes, a second heat map was generated from the differences in each plane's termination point from its location in the previous video frame; in other words, the velocity of each plane in each frame is presented in Figure 4.11. Examination of the frame-to-frame variations shows that all of the $\{111\}$ planes along the interface were observed to undergo both grow and retraction events.

Focusing on the larger velocities, events with a magnitude greater than $5.6 \AA \cdot \sec ^{-1}$ were defined to be significant; this value corresponds a two $\sigma$ event in the Gaussian distribution. Virtually all of the significant positive (growth) and negative (dissolution) velocities were immediately accompanied by a complimentary event in the opposite direction in the following time-step. This tendency to immediately reverse significant changes is indicative of the system being at, or extremely close to, the equilibrium state. A few events resulted in significant oscillations between relatively large positive and negative velocities that required more than three time-steps to damp back toward zero. For example, beginning in frame 27 and continuing through frame 32, plane number 3 accelerates sharply in both the positive and negative directions with velocities of $+10,-10$, $+7,-5,+1$, and $-6 \AA \cdot \sec ^{-1}$. 


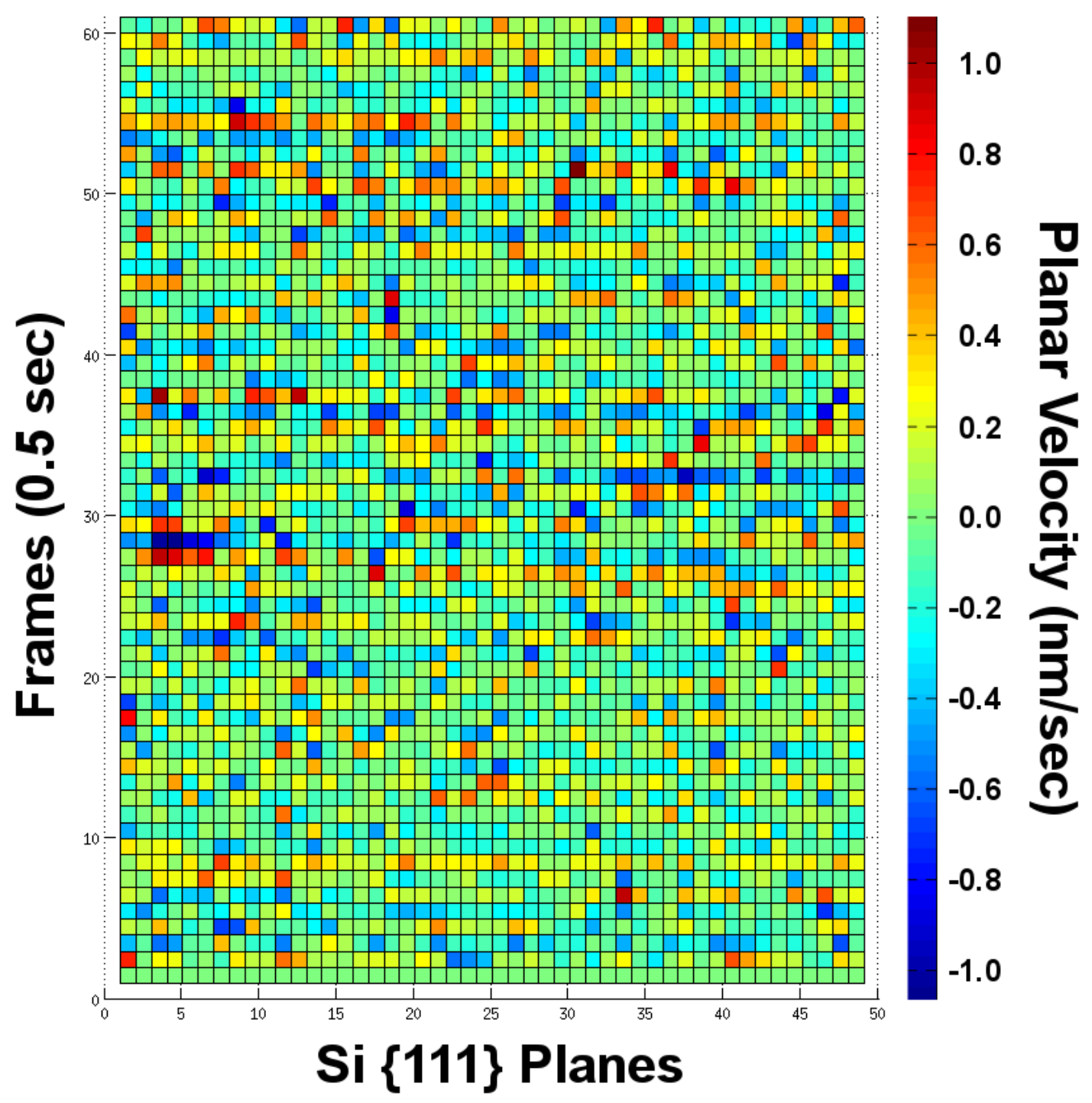

Figure 4.11: A heat map used to represent the frame-to-frame differences of the $\{111\}$ plane lengths along the interface for a period of 30 seconds.

The distribution of significant motion of individual $\{111\}$ planes and groups of adjacent $\{111\}$ planes, shows approximately an equal number of events that began with both positive and negative velocities. There was no correlation between the beginning of these events along either interfacial positions nor the direction of the local interfacial motion.

After combining every frame-to-frame change into a single dataset, a histogram was plotted to show the distribution of all changes in planar lengths over the entire time period, shown above in Figure 4.12. The entire dataset is centered about zero with virtually equal areas in the positive and negative directions. Two 


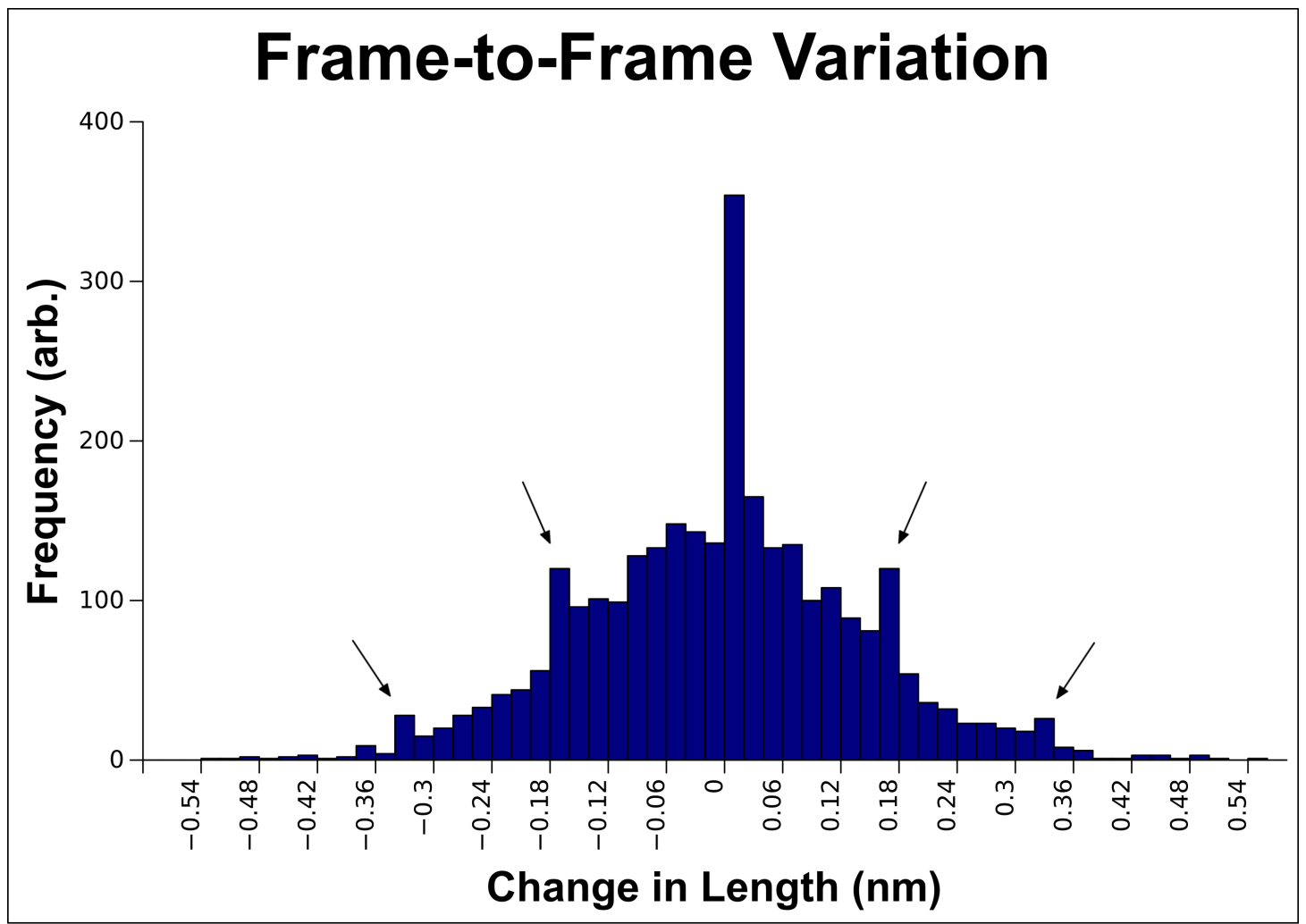

Figure 4.12: A histogram generated from the frame-to-frame variations plotted in Figure 4.11 showing near zero net change with the characteristic changes in length indicated by the arrows.

characteristic spacings are evident in both the positive and negative directions; these are within the histogram bins of $\pm 1.8-1.6 \AA$ and $\pm 3.4-3.2 \AA$. The peak, indicated by the arrows in Figure 4.12, at $\pm 3.4-3.2 \AA$, is a sum-peak resulting from two near simultaneous growth events of 1.6-1.7 $\AA$. Calculation of a number of lowindex interplanar spacings resulted in a single match; the $\{113\}$ interplanar spacing in crystalline silicon at $650{ }^{\circ} \mathrm{C}$ is $1.64 \AA$. Thus, from analysis of the composite dataset, it is evident that both the growth and dissolution events at the interface were driven towards moving in increments of the $\{113\}$ interplanar spacing. In addition to this characteristic length (or amplitude of movement), a large number of non-characteristic changes occurred and they fit a roughly Gaussian distribution with a standard deviation of $1.4 \AA$. Both Gaussian and Lorentzian fits to the noncharacteristic data were explored. The Gaussian distribution fit the central region better while the Lorentzian distribution was better at characterizing the data at the edges of the histogram; the difference between the $\mathrm{R}^{2}$ values was less than 0.02 . From the fits, it is likely that these data are best described by a Voigt function 
which convolutes these two statistical distributions.

Previous theoretical calculations on metal alloys have shown the distribution planar height changes to be Gaussian ${ }^{22}$. In this previous investigation, based on a hard sphere potential, no bias in the magnitude of the fluctuations occurred towards crystallographically related values. The observations reported here of the bias towards quantization magnitudes of fluctuations relating to the $\{113\}$ interplanar spacings, are likely caused by the strong directional-nature of the covalent bonding present in crystalline silicon. These data, coupled with additional atomistic modeling, may provide further insight into fundamental processes occurring at the solid/liquid interface during both equilibrium capillary fluctuations and during solidification processes.

\subsection{Heterogeneous Nucleation of Copper on the Silicon $\{113\}$ Solid/Liquid Interface}

\subsubsection{Experimental Observation}

Throughout the period of observation of the dynamic behavior present along the solid/liquid $\{113\}$ interface, a weak periodic oscillation of intensity was intermittently observed in varying locations along the length of the interphase boundary. An extracted series of frames exhibiting this phenomena are shown in Figure 4.13, and a further enlargement is shown in Figure 4.15b. Figures 4.13be show consequetive frames in which these osciallations are highlighted with increased contrast to aid visualization, while Figure 4.13e shows an unmodified frame. The transient nature of this spatial periodicity, coupled with the fact that the phenomena only occurred along a portion of the field of view, led to the conclusion that rather than being a contrast delocalization effect, this phenomena was caused by the transient presence of an unknown phase at the solid/liquid interface. 
The periodic spacing of this transient phase was measured to be approximately $1.7 \AA$. Based on this data, and the interplanar spacings of possible phases within the alloy, the interfacial phase was determined to most likely be copper. The observed periodicity matches copper's $\{200\}$ interplanar spacing of $1.8 \AA$ to within $6 \%$, which, at this magnification, is within the accuracy of the HRTEM image spatial calibration. Additionally, experimental evidence of the segregation of copper to this solid/liquid interface has been reported in the same aluminum alloy ${ }^{35,45}$.

\subsubsection{Determination of the Structure of the Copper-Silicon Solid/Solid Interface}

Interfaces between crystalline solids are frequently characterized by orientation relationships present between the two phases across the interface ${ }^{6}$. Based on the known crystallographic orientation of the silicon and the observation of only the copper $\{200\}$ planes and their angle relative to the silicon, the orientation relationship of this solid/solid could be determined. The copper $\{200\}$ planes are oriented $148^{\circ}$ counter-clockwise from the silicon $\{111\}$ planes. Given these orientation criteria, the stereographic projection of copper was used to search for potential interfacial planes. Candidates for copper's interfacial plane were then evaluated with the atom-row matching $\operatorname{method}^{47,48}$.

The atom-row matching method is commonly used to evaluate interfaces between crystalline solids. According to the theory, stable interfaces are formed when the number of overlapping atom-rows on either side of the solid/solid interface is maximized. It has been shown that maximizing the overlap of individual atomic sites between the crystalline faces is less important than maximizing the overlap between atom-rows when determining and/or predicting the structure of solid/solid interfaces ${ }^{49}$.

The close-packed directions along each lattice's interfacial plane were aligned and atom-row positions within a $15 \%$ spatial tolerance were sought. Utilizing this 


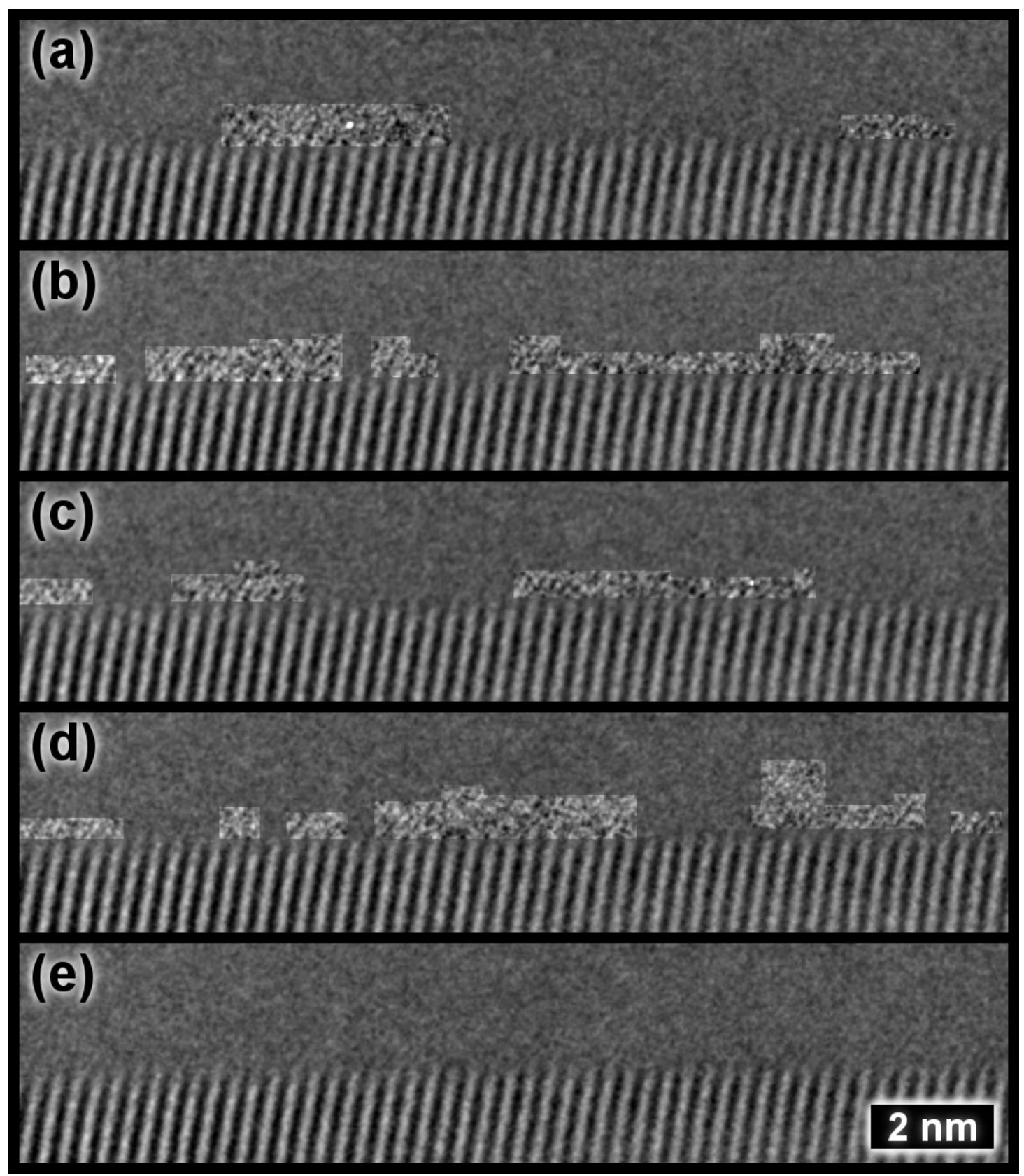

Figure 4.13: (a-d) A tableau of consecutive frames of video showing copper nuclei forming at the solid/liquid interface. The locations of the copper nuclei have been selectively contrast-enhanced in order to aid identification in this document; (e) the as recorded frame shown in (d). 


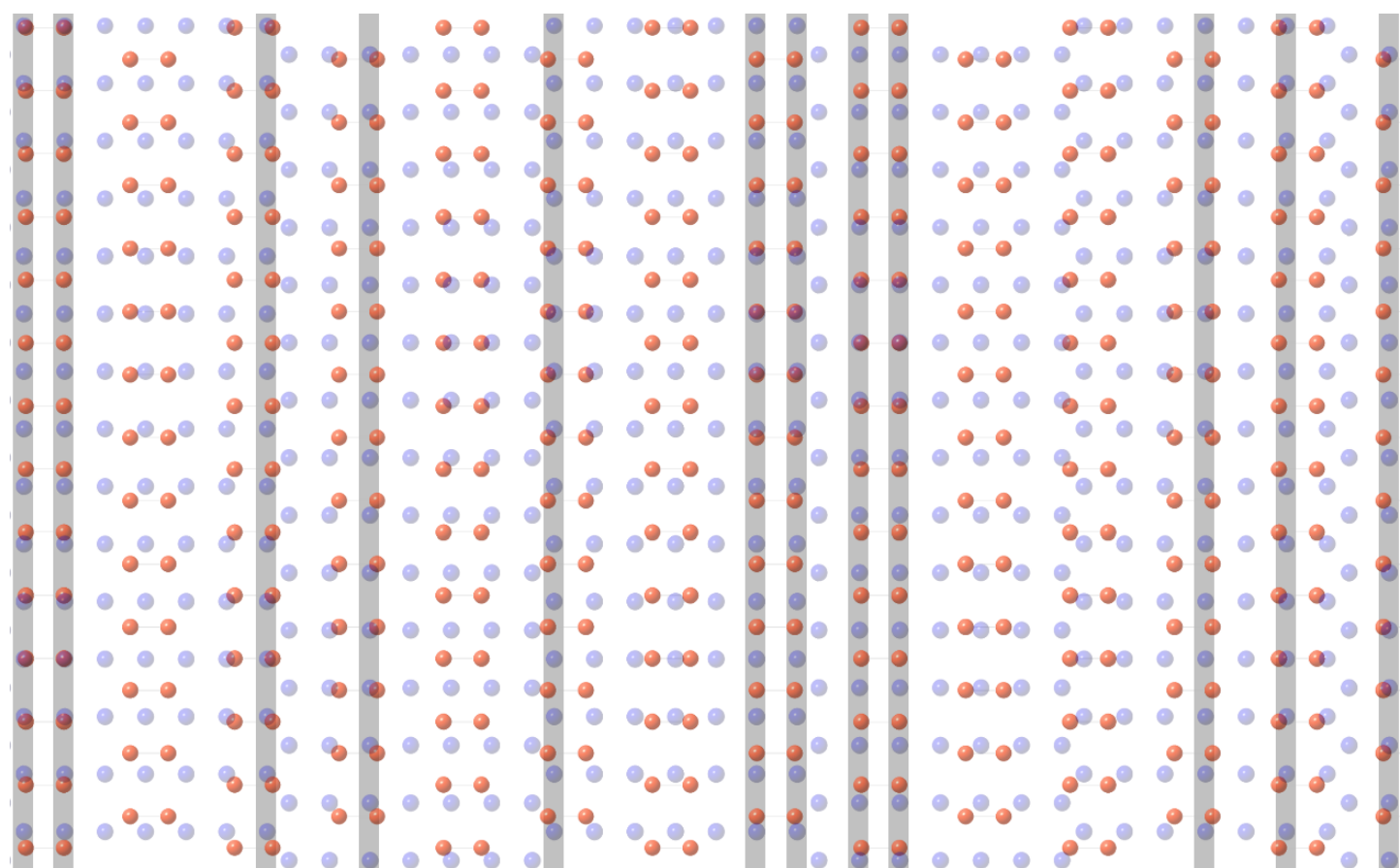

Figure 4.14: A schematic depicting the atom-row matching between silicon $\{113\}$ atomic sites, in orange, and copper $\{076\}$ atomic positions, in blue. Vertical rows matching within $\pm 15 \%$ are highlighted.

method, a number of possible copper interfacial planes were evaluated. Figure 4.14 shows a copper $\{076\}$ plane superimposed onto the silicon $\{113\}$ interfacial plane; the atom positions are represented in blue and orange, respectively. With the closepacked directions aligned vertically, the number of rows within the $15 \%$ spatial tolerance were determined. In Figure 4.14, 12 of the 27 silicon rows fall within this criterion or a $44 \%$ match. Expanding the measured area to approximately four times the size shown in the figure in order to minimize any potential selection bias results in this interfacial configuration matching along $42 \%$ of the silicon atomrows. This interfacial configuration was determined to be the best match of all possibilities explored.

\subsubsection{High-Resolution TEM Image Simulation}

Confirmation of the proposed solid/solid orientation relationship was conducted through the use of high-resolution TEM image simulation. A threedimensional model of the interface was constructed using the Crystal Kit software 
package (Total Resolution LLC., Berkeley, CA). The solid/solid interfacial model was constructed by allowing a copper crystal's (076) facet to penetrate $1 \AA$ into the silicon lattice's (113) plane. Then all atoms within $1 \AA$ of their nearest neighbors were removed to prevent an unphysical over-occupation. The copper lattice beyond a distance of $5 \AA$ off the silicon crystal was removed. Finally a super-cell $34 \AA$ wide by $65 \AA$ tall and $20 \AA$ thick was defined; this solid/solid/vacuum super-cell was then imported into the MacTempas software package (Total Resolution LLC., Berkeley, CA) to simulate HRTEM images.

Due to complications in the process of simulating HRTEM images with accurate atomic structures of liquids, the solid/solid/vacuum heterostucture was used. The purpose of this image simulation process was to explore and confirm the solid silicon/solid copper interfacial structure, and the contrast oscillations present in either the vacuum or the liquid were not of interest, and so were not included.

Within MacTempas, the simulation parameters were set up to duplicate the microscope used throughout this series of experiments. The FEI SuperTwin objective lens has a spherical aberration coefficient of $1.2 \mathrm{~mm}$. The beam convergence angle and spread of defocus during imaging were estimated to be 0.7 milliradians and $70 \AA$, respectively. These estimations were made by comparing the FFT of a thick amorphous carbon film with the calculated contrast transfer functions generated within MacTempas. During imaging of the copper islands, the defocus was estimated to be $-60 \mathrm{~nm}$. The crystal tilt relative to the silicon lattice was estimated to be approximately 30 milliradians along the visible $\{111\}$ planes. Finally, the super-cell structure was sliced into $2 \AA$ thick phase-gratings for the simulation process.

The output image from the simulation process was overlaid onto an experimental image. The simulated intensity from the vacuum region was removed to allow the experimental image's liquid phase region to be observed with the simulated structure. The resulting comparison between the experimental and simulated images is shown in Figure 4.15, where excellent agreement is seen. 


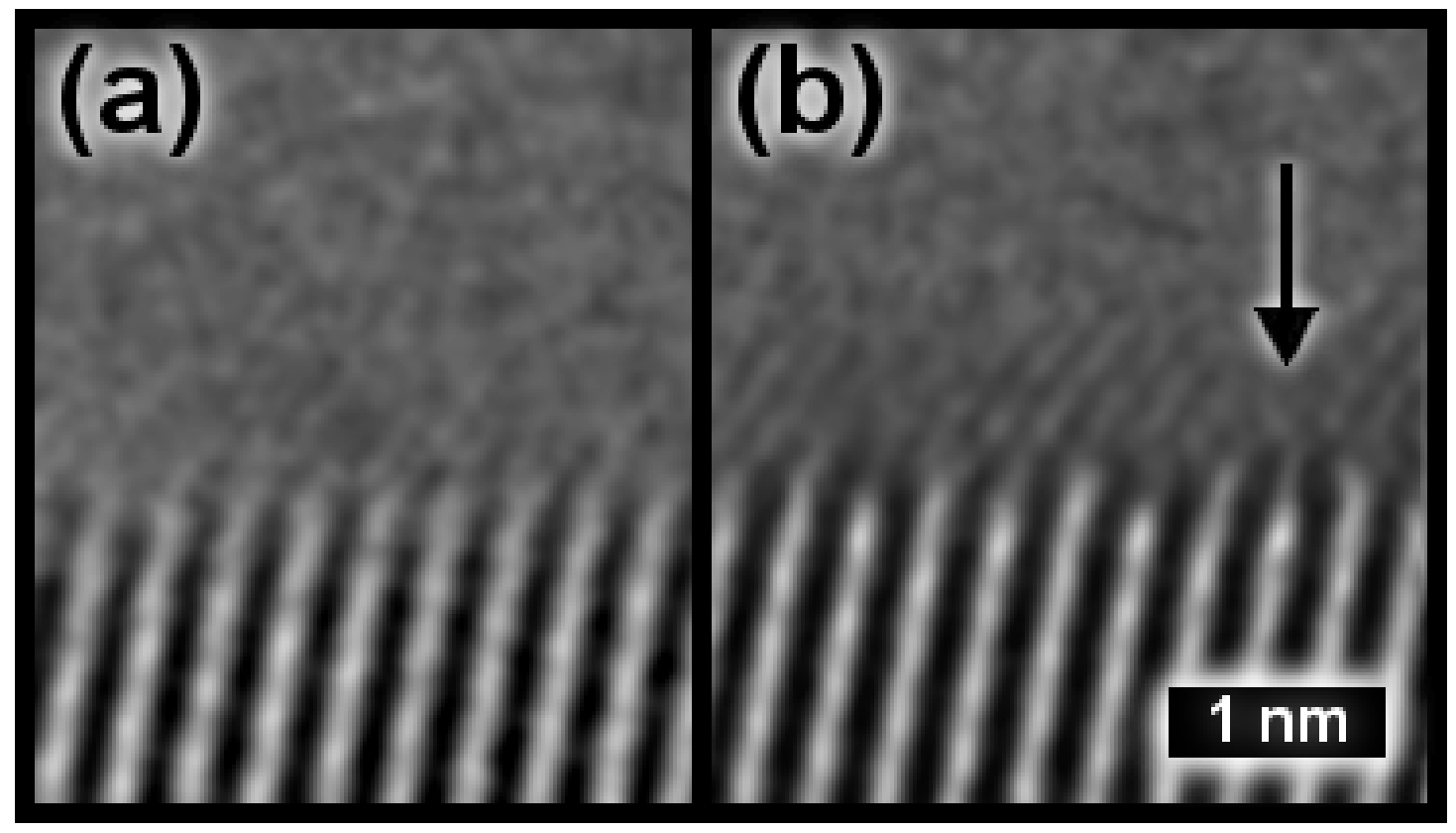

Figure 4.15: Comparison of the experimentally observed high-resolution image (a), and the simulated image (b) of copper (076) on the silicon (113) solid/liquid interface; the arrow highlights two silicon $\{111\}$ planes appearing to merge into a surface reconstruction.

The intensity oscillations from the copper layer in the simulated image are stronger than the experimental micrograph. This difference is due to the variation in thickness of the copper along the viewing direction. The simulation was performed with copper through the entire 20-nm-thick simulation cell. The experimental image intensity oscillations are likely being damped by the presence of the overlapping liquid phase above and/or below the copper patches present on the interface, since they do not extend through the entirety of the sample thickness along the viewing direction.

Based on the disappearing and reappearing nature of the small copper islands along the interface, previous reports of copper segregation prior to the nucleation of the $\theta$-phase ${ }^{35,45}$, and the fact that the interface was at or very near equilibrium, the copper islands were most likely interfacially stabilized nuclei of a copper precursor phase forming at the solid/liquid interface. The dynamic behavior of the solid silicon at this solid/liquid interface may be refelcted in the transient presence of these copper patches.

In Figure 4.15b, an arrow on the right-hand side points to two silicon $\{111\}$ 
planes that appear to merge together. This phenomenon was observed in several locations along the solid/liquid interface in numerous frames. The appearance of this was initially interpreted as an unknown type of silicon surface reconstruction occurring on the $\{113\}$ interfacial plane. The high-resolution simulation of the copper nuclei revealed that this phenomenon occurs without a reconstructed structure present in the input file used for the simulation. This leads to the conclusion that the copper/silicon interaction is responsible for the appearance of this effect, and it may be related to the misfit between the $\{200\}$ copper planes and the $\{113\}$ silicon planes meeting at the interface.

\subsubsection{Characterization of the Copper Nuclei}

After identifying the origin of these intensity oscillations at the solid/liquid interface due to copper, and exploring the structure of the solid/solid interface, quantification of the dimensions of the fluctuating patches of copper was conducted. Throughout the 61 frames of video, 206 copper islands were measured (e.g. Figure 4.13). For each observed copper island, the length along the solid/liquid interface and the height extending off of the crystalline silicon surface were measured and recorded. Histograms of each measured quantity were plotted in Figures 4.16 and 4.17.

As shown in Figure 4.16, the distribution of heights of the copper islands was approximately Gaussian in nature. The 206 islands, on average, extended $5.6 \AA$ off of the silicon surface with a standard deviation of $1.9 \AA$. The maximum height observed was $13 \AA$. In contrast to the relatively small dimensions of island heights, the sub-critical nuclei lengths along the interface were observed to follow a lognormal distribution, as shown in Figure 4.17. This distribution of individual island widths spanned from a single pair of adjacent copper $\{200\}$ planes with a length of $2 \AA$ all the way up to an island $130 \AA$ wide. The strong skew in these data towards smaller island widths has been observed in a number of nucleation and growth studies $^{5,50}$. This distribution is understood to be a result of a combination of high 


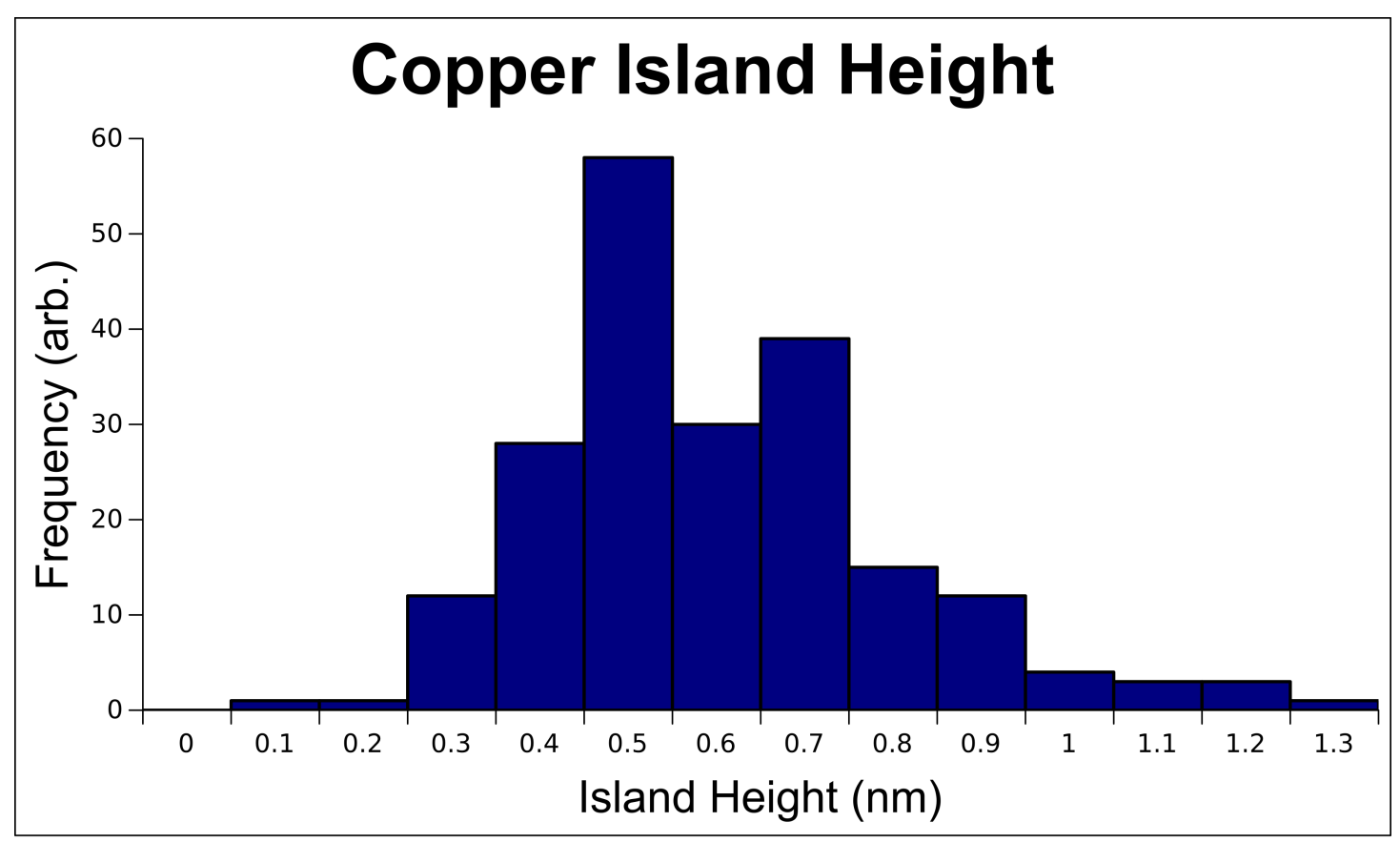

Figure 4.16: A histogram showing the distribution of the heights that the copper nuclei on the solid/liquid interface penetrated into the liquid phase

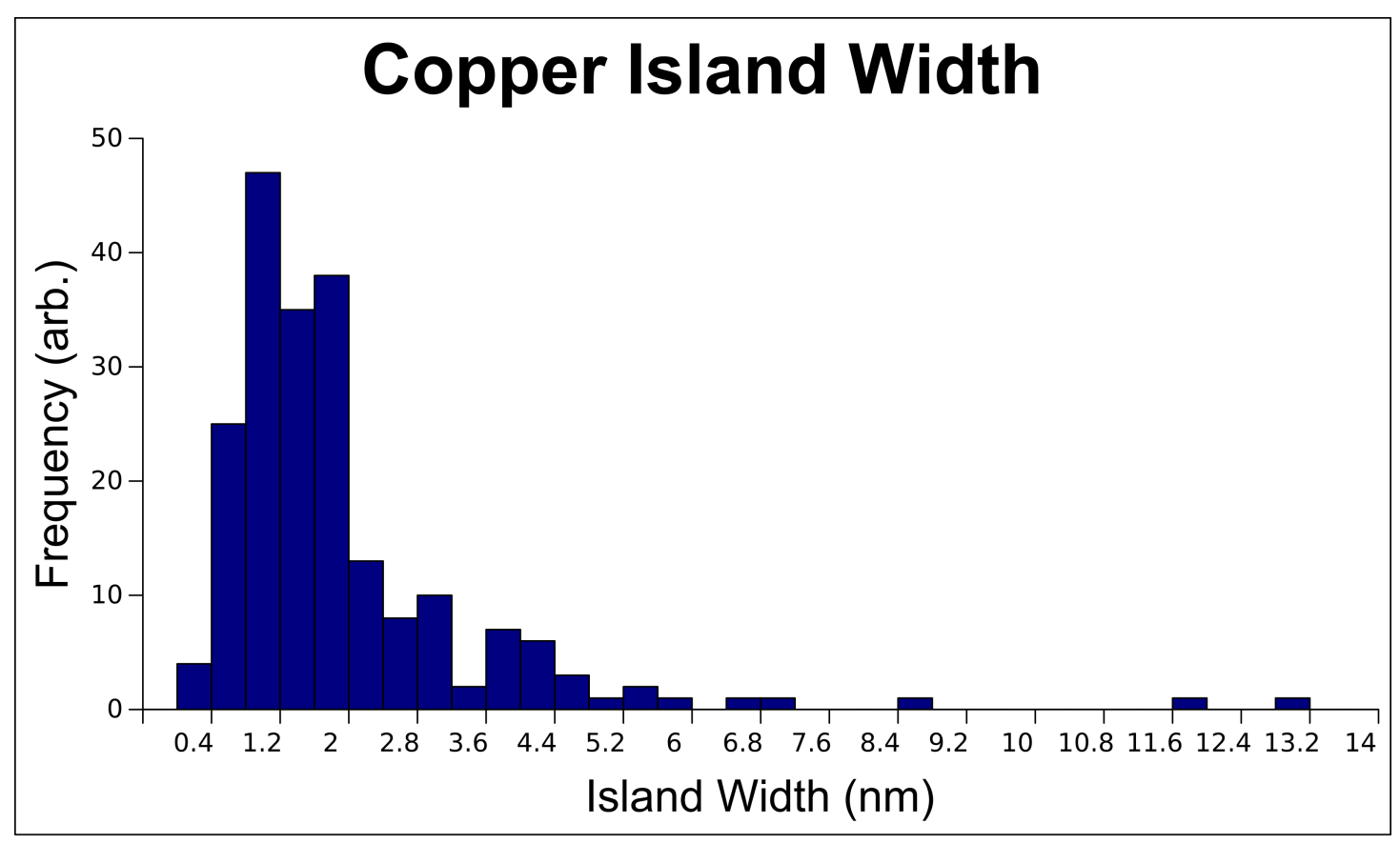

Figure 4.17: A histogram showing the distribution of the widths of the copper nuclei observed along the solid/liquid interface 


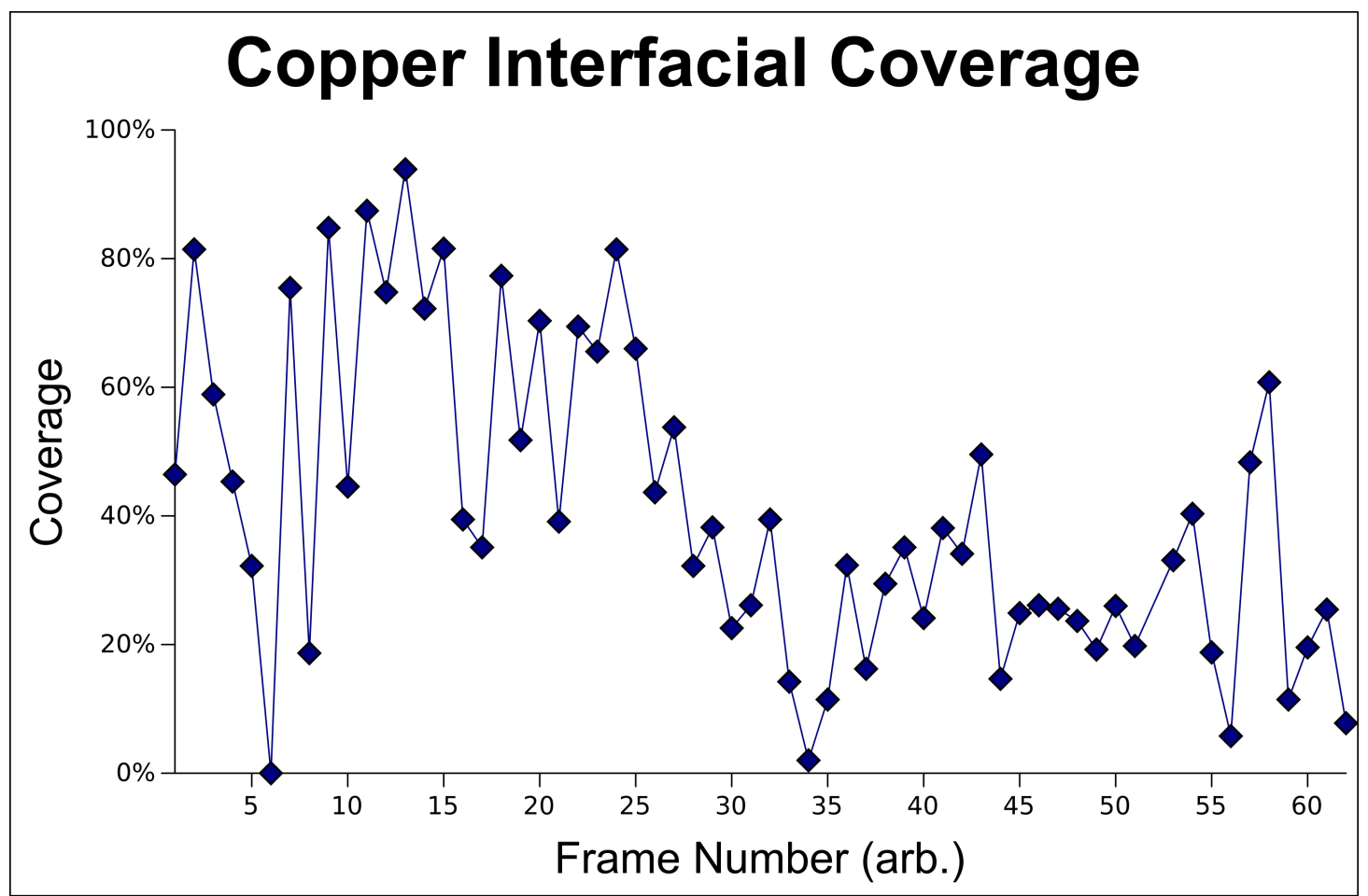

Figure 4.18: A plot of the percentage of the solid/liquid interface covered by copper islands within a given frame.

surface mobility and the coalescence of small islands driven by the minimization of surface free energy.

The relative coverage of the solid/liquid interface also varied, along with the large variability of the copper island heights and widths. Figure 4.18 shows the percentage of the length of each frame's interface covered by the crystalline copper phase. Overall, there is a trend in the plot towards decreasing coverage over time, i.e. the interface was $\sim 50 \%$ covered in the first half of the observation period and this decreased to $\sim 20 \%$ in the latter half, however there aren't enough data to determine if this trend is long-term or just a statistical variation.

Correlating the coverage of copper with the motion of the solid/liquid interface shown in Figure 4.11 shows evidence of the copper being able to suppress the dynamic behavior of the interface. Frames 11-15 show less motion of the silicon planes at the interface than average, while this series of frames are $\sim 80 \%$ covered in copper. Conversely, frames 45-52 underwent larger than average motions of the silicon $\{111\}$ planes at the interface and were $\sim 25 \%$ covered by copper. In 
frames $24-34$, there is a large decrease in the copper coverage evident and a large increase in the amount of motion of the silicon interface. The presence of copper hindered the motion of the silicon planes in both the positive (growth) and negative (dissolution) directions.

Despite the rough nature of the silicon $\{113\}$ solid/liquid interface, both the nuclei size distribution as well as the transient nature of individual islands are indicative of interfacial diffusion readily occurring at the interface. Even though the $\{113\}$ interfacial plane presumably has a series of troughs running along the $<110>$ viewing direction like a $\{113\}$ silicon surface ${ }^{51}$, lateral diffusion must occur in order to see the migration of copper from the viewing direction in these experiments. The temporal resolution is insufficient to determine if the copper islands were migrating laterally, or if they were forming and dissolving rapidly. In either case, the nucleation and growth of the copper islands on the interface between the solid silicon and the liquid alloy resulted in either a metastable phase, or sub-critically sized nuclei, of a phase that precedes the nucleation of $\epsilon_{2}$-phase or other copper-rich precipitates like $\theta$-phase observed along the interface ${ }^{45}$.

In a review, Spaepen states ${ }^{17}$ "...the interface as a region with its own thermodynamic parameters does not imply that it is being treated as a separate phase. It can only exist between two bulk phases, and its thermodynamic properties only have meaning as transition parameters between the bulk values." It is within this transition layer, i.e. within $1 \mathrm{~nm}$ of the solid crystal surface, that the observation of the copper islands are made. On the equilibrium phase diagram, Figure 4.3, solid copper should not begin to form until concentrations on the order of $\sim 85 \%$ are reached.

Based on its continuous, but rapidly fluctuating presence, the formation of copper is arguably energetically favorable in the unique thermodynamic environment that exists at this solid/liquid interface. The orientation relationship consistently observed between the copper nuclei and the silicon substrate maximizes the number of bonds between the silicon and copper phases. While it is not possible with the given dataset to quantify the exact ratio of broken 
bonds between the crystalline silicon and copper due to the complexity of the interface, the presence of this phase suggests that it is energetically favorable to form copper/aluminum-rich liquid bonds rather than leaving the aluminum liquid/silicon interface free from the presence of this phase.

As a rough estimate, it is instructive to take the cohesive energies of each atomic species divided by the number of nearest neighbors per atom to estimate the excess energy per broken bond. Silicon and has a cohesive energy of $4.63 \mathrm{eV}$ per atom $^{52}$ and has four nearest neighbors, $1.16 \mathrm{eV}$ per bond; copper has a cohesive energy of $3.49 \mathrm{eV}$ per atom ${ }^{52}$ and twelve nearest neighbors, $0.29 \mathrm{eV}$ per bond. With the varying facets and varying areas exposed to the liquid, it is ultimately the number of dangling bonds per area at each solid/liquid interface which must be examined to properly account for the full understanding of these trade-offs. In this environment, copper nuclei, at least temporarily, act as a transition between the high energy penalty of a broken silicon bond and the ability of the liquid to satisfy these bonds.

In previous reports of copper segregating to the solid/liquid interface in this alloy system ${ }^{35,45}$, the amount of copper enrichment was statistically significant, but small, on the order 0.2 at. \%. In both of these reports, the EDS signal was collected from an electron probe $25 \mathrm{~nm}$ in diameter, and was assumed that copper was spread evenly across the interface, perhaps as monolayer segregation. The present results suggest that it is possible that the previous reports were due to the same phenomenon occurring, i.e. the formation of fluctuating copper islands, but the presence of the copper islands wasn't observed due to the limited spatial resolution of the microscope employed.

In these same studies, $\theta$-phase $\mathrm{Al}_{2} \mathrm{Cu}$ was reported to nucleate and grow on an unknown high-index facet ${ }^{45}$. The presence of the islands of copper presented here potentially aided the nucleation and growth of the $\epsilon_{2}$ phase when it grew off of the solid silicon/liquid alloy interface. The previously observed segregation of copper, if, in fact, it was the same phenomenon likely have played a role in the nucleation of $\theta$-phase observed. 


\section{Chapter 5}

\section{Summary and Conclusions}

During the experimental process, a significant increase in copper concentration within the nanoparticles was measured. The source of this copper was likely surface diffusion along the amorphous carbon film emanating from the TEM grid itself. The AA390 alloy nanoparticles were shown to readily absorb this copper, as evidenced by a depletion zone around alloy nanoparticles imaged with EFTEM imaging. The substantial increase in copper concentration resulted in hetergeneous nucleation of $\epsilon_{2}$-phase precipitates at the solid/liquid interface between either the solidified silicon or along the inner surface of the oxide shell. A balance between the amount of copper coming into the ally nanoparticle studied, and the growth of the copper-rich $\epsilon_{2}$-phase, provided a stead-state situation, i.e. local equilibrium, at the solid/liquid interface, as evidenced by the stationary interface position with time at $650^{\circ} \mathrm{C}$.

The equilibrium dynamic behavior of the interfaces near solid/liquid/vapor triple point were studied at $650{ }^{\circ} \mathrm{C}$ over a period of ten seconds by in situ HRTEM. Frame-by-frame analysis of the video recording showed patterns of behavior present at the interface between the solid silicon and liquid-phase aluminum alloy. Three different areas/topics were analyzed in detail: (1) The motion of steps in $\{111\}$ planes as the solid/liquid interface curved away from the triple-point with the vapor, (2) Motion of planes comprising a flat $\{113\}$ solid/liquid interface, and 
(3) formation of a metastable copper phase forming at the $\{113\}$ interface. Results and conclusions from each of these three areas/topics are summarized below.

\subsubsection{Motion of $\{111\}$ Planes at the Triple-Point}

- The interface between the solid silicon/liquid aluminum alloy constantly fluctuated between a rough interfacial morphology and either a smoothly faceted or a smoothly rounded structure. Highly faceted configurations, with up to four distinct crystallographic facets, were observed as the stable solid/vapor (111) interface transitioned through the triple point to the stable (11̄3) solid/liquid interface farther back in the particle.

- The stability of the various interfacial morphologies showed that only faceted configurations persisted from frame-to-frame; neither the smoothly rounded nor roughened solid/liquid interfacial structures were stable for more than a single 0.5-second time-step. The observed stability of the faceted configurations is indicative of their lower interfacial free energies relative to either the roughened or the rounded interfacial morphologies; this result is consistent with the highly anisotropic surface free energies of silicon.

- Volumes of crystalline silicon exhibiting weaker phase-contrast at the solid/liquid interface were shown to solidify and dissolve rapidly. From a similar contrast attenuation of the slightly off-axis silicon $\{111\}$ planes and the frame-to-frame persistence of one of the silicon $\{111\}$ planes exhibiting this phenomenon, it was concluded that rather than capturing a rapid solidification/dissolution event that occurred faster than the exposure time of a single frame, this behavior was consistent with silicon solidifying through only a portion of thickness along the viewing direction. These volumes with weaker contrast were most likely caused by pill-box-type nucleation events extending off of the solid/liquid interface through only a fraction of the sample thickness. 


\subsection{6 $\{113\}$ Solid/Liquid Interfacial Behavior}

- The dynamic equilibrium behavior of the interface between a high-index facet on the primary silicon particle and the liquid phase was studied with high spatial resolution at $650{ }^{\circ} \mathrm{C}$. Along the $\{113\}$ facet of the silicon crystal, the $\{111\}$ planes were constantly fluctuating in length. The change in length of 49 planes was tracked through 61 frames, and these changes were examined in detail.

- Through the use of one-dimensional FFTs, there was no correlation between the location of individual and/or groups of $\{111\}$ planes undergoing growth and/or dissolution events either along the interface or through the temporal dimension. This was found to be in contrast with previous studies ${ }^{28}$ of incoherent boundaries at solid/solid interfaces.

- Looking at the entire set of frame-to-frame variations in planar length, it was found that there was a tendency for the silicon $\{111\}$ planes to extend and retract by quantized intervals of length corresponding to one and two times the $\{113\}$ interplanar spacing within the silicon lattice. This bias was superimposed on a Gaussian distribution of the changes in lengths; while the Gaussian distribution matches previous computational models ${ }^{22}$, the bias towards crystallographically related changes in length is reported as a new finding.

\subsubsection{Copper Island Observation and Characterization}

- The heterogeneous nucleation an unknown phase was observed on the interface between the solid silicon and liquid-phase aluminum alloy. Small islands of this phase were present transiently along the interface. Large frame-to-frame variations in island length along the interface as well as in the percentage of interfacial coverage were observed.

- Based on the $1.7 \AA$ lattice spacing of this phase, the phases likely present within the system, and prior reports of segregation of copper to the 
solid/liquid interface ${ }^{35,45}$, it was determined that the spacing matched the copper $\{200\}$ interplanar spacings. Thus, it was concluded that the copper islands form at the solid/liquid interface, presumably as sub-critical nuclei, precursor phase prior to nucleation of an equilibrium copper-rich phase.

- HRTEM image simulation was used confirm the structure of the interface and the $(1 \overline{1} 3)_{S i}\left\|(076)_{C u} ;[110]_{S i}\right\|(100)_{C u}$ orientation relationship between the solid phases. The atom-row matching method was employed and potential orientation relationships between the solid silicon and solid copper phases were examined and it was found that $44 \%$ of the silicon atom-rows aligned with copper in this orientation relationship.

- The copper islands, while varying widely in length along the solid/liquid interface, never extended more than $1.3 \mathrm{~nm}$ into the liquid. The heights displayed a Gaussian distribution while the lengths were log-normal, and these are characteristic for a process where rapid diffusion and coalescence are present at an interface.

\section{$5.1 \quad$ Future Work}

Recent advances in both direct electron detection cameras and in situ heating holders will enable significantly more detailed study of solid/liquid interfaces. With the recent advent of the commercialization of ultra-high speed direct electron detection cameras, time-scales on the order of $1 / 1600^{\text {th }}$ of a second are now accessible. The transitory process of individual atoms or groups of atoms from the liquid to solid states may yet reveal further underlying physics which have yet to be illuminated at atomic-resolutions on such short time-scales.

In addition to the new temporal regimes experimentally accessible, next generation in situ heating holders have been developed using the advances in microelectromechanical systems (MEMS) based technologies. These holders, rather than using a bulk furnace to heat up a relatively large thermal mass, instead 
highly localize the heating process to a very limited sample volume. One major advantage of such a design is a large reduction in thermal drift; in this work the thermal drift from the sample holder was found to limit the period of observation. As an additional benefit, with the large reduction in thermal mass, the use of EDS at elevated temperatures is possible without the need for the installation of a thermal shield. In addition to potentially enabling further detailed EDS analysis, the high degree of thermo-mechanical stability at experimental temperatures enables EFTEM imaging at increasingly higher magnification ranges, further increasing the spatial resolution of this powerful elemental mapping technique.

The process of continual adsorption of copper from the TEM grid, coupled with improvements in chemical analyses, holds promise of new insights into alloying processes. Time-resolved changes in the chemistry and distributions of atomic species may further illuminate the process of solute segregation to solid/liquid interfaces. 


\section{Appendix A}

\section{MATLAB Algorithm}

\section{'measuremement.m'}

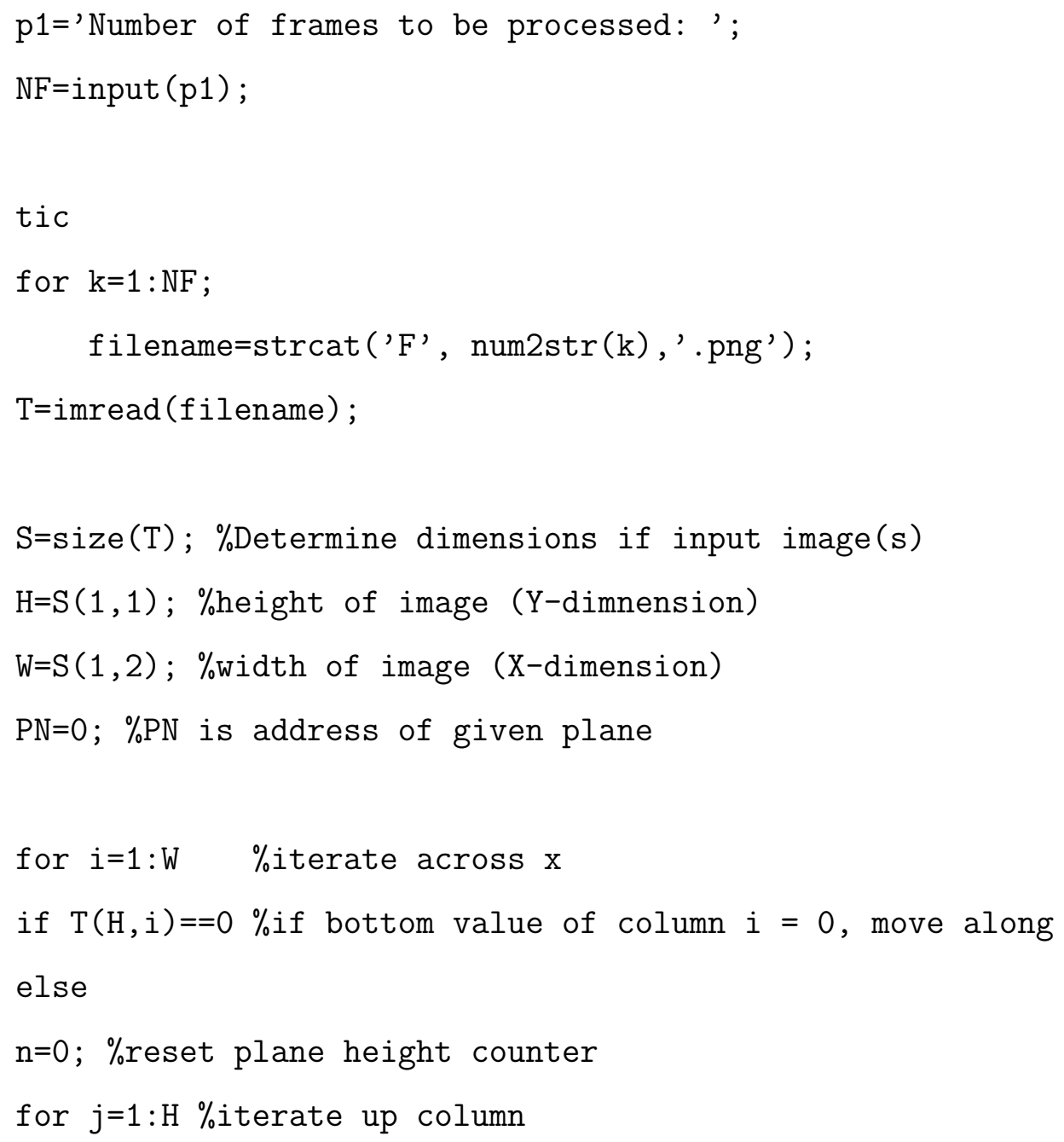




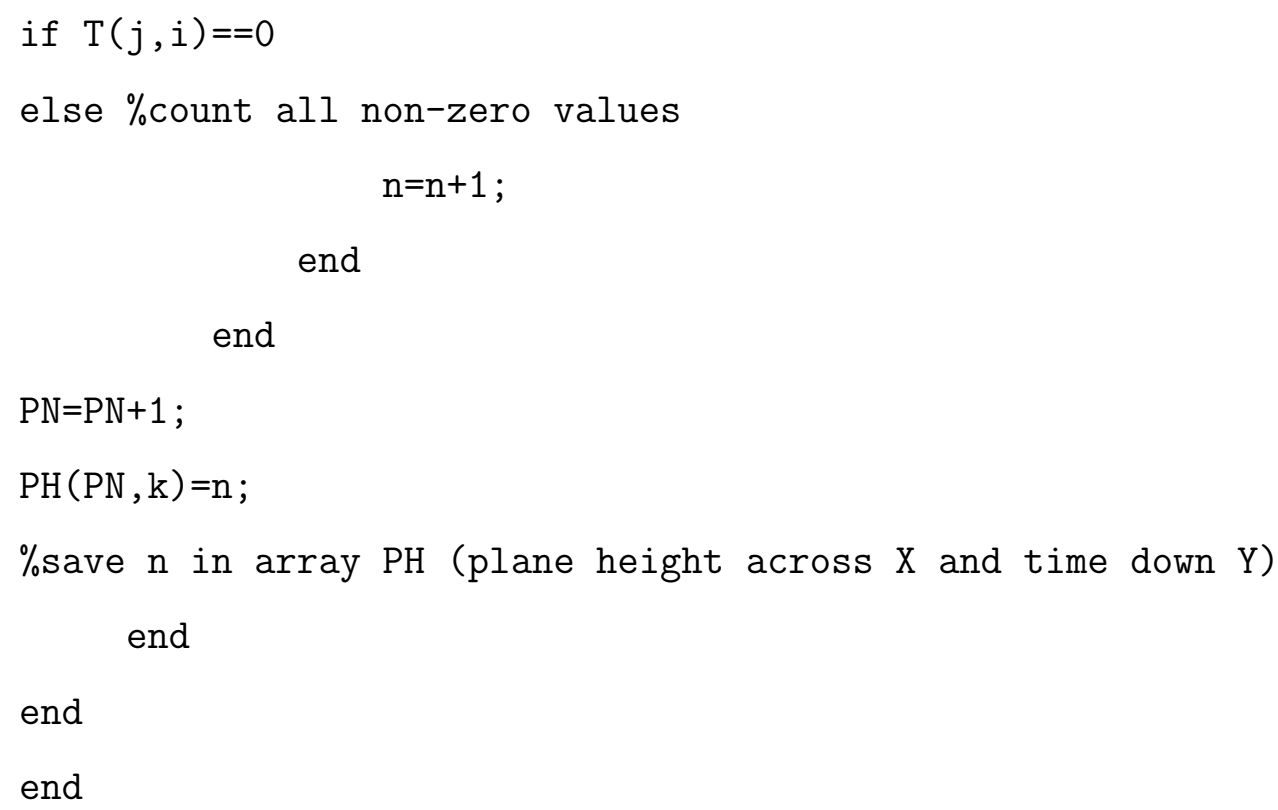


G_stats=[global_max, global_min, global_avg, global_std] ;

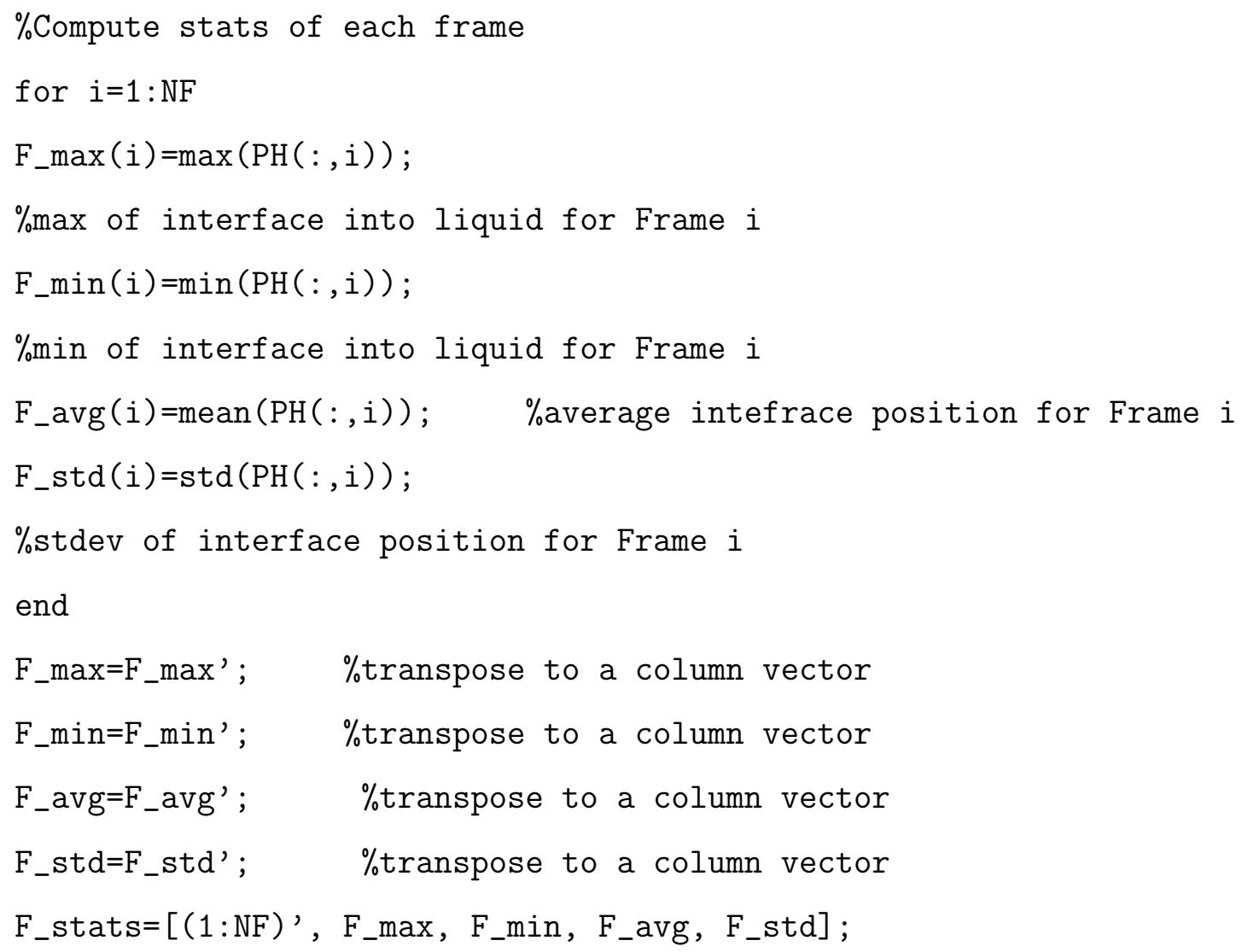


$\operatorname{Delta}(i, j+1)=D$;

end

end

\%Look at all planes above/below average interface location for $i=1: N F$;

$F_{-} \operatorname{aAvg}(:, i)=P H(:, i)-F \_a v g(i)$;

$\%$ subtract avg in frame $i$ from each plane

for $j=1: R$; \%go through each value for frame $i$

if $F_{-} \operatorname{aAvg}(j, i)<0==1$;

$\%$ check to see if plane position is less than zero

$F_{-} b \operatorname{Avg}(j, i)=-1 . * F_{-} \operatorname{aAvg}(j, i) ; \%$ if $>$ zero, add below average values

$F_{-} \operatorname{aAvg}(j, i)=0 ; \%$ set value to zero in the above average array

end

end

end

$\%$ In each frame, look at all planes 1 stdev above average for $i=1: N F$;

F_aStd $(:, i)=F_{-} \operatorname{aAvg}(:, i)-F_{-} s t d(i) ; \%$ subtract stdev from >avg planes for $j=1: R$; \%go through each value for Frame $i$

if $F_{-} \operatorname{aStd}(j, i)<0==1$; $\%$ is plane position is less than zero?

$F_{\text {aStd }}(j, i)=0 ; \%$ if it is, set to zero

end

end

end 
\%In each frame, look at all planes 1 stdev below average for $i=1: N F$;

F_bStd $(:, i)=F_{\text {_bAvg }}(:, i)-F_{-}$std $(i)$; \%subtract stdev from <avg planes for $j=1: R$; \%go through each value for Frame $i$

if $F_{-} b \operatorname{Std}(j, i)<0==1$; $\%$ is plane position is less than zero?

F_bStd $(j, i)=0 ; \%$ if it is, set to zero

end

end

end

$\%$ compute net changes across all time

for $i=1: R$

$\operatorname{net}(i)=\operatorname{sum}(\operatorname{Delta}(i,:))$;

end

net=net'; \%transpose to a column vector

\%compute "thickness" of interface

height_max=max $(\mathrm{PH})$;

height_min=min (PH) ;

thickness=height_max-height_min;

thickness_avg=mean (thickness);

thickness_std=std (thickness); 
$\%$ compute FFT's of the S-L interface frame-by-frame

for $i=1: N F$;

PH_FT $(:, i)=f f t(P H(:, i))$;

end;

PH_FTCC $=$ conj $($ PH_FT) ;

PH_FFT $=$ PH_FT.$*$ PH_FTCC ;

\%compute FFT's of above-averge S-L interface frame-by-frame for $i=1: N F$;

F_aAvg_FT $(:, \dot{i})=f f t\left(F_{-} \operatorname{aAvg}(:, i)\right)$;

end ;

F_aAvg_FTCC $=\operatorname{conj}\left(F_{-}\right.$aAvg_FT $)$;

F_aAvg_FFT=F_aAvg_FTCC. $*$ F_aAvg_FT;

\%compute FFT's of below-averge S-L interface frame-by-frame for $i=1: N F$;

F_bAvg_FT $(:, \dot{i})=f f t\left(F \_b A v g(:, i)\right)$;

end;

F_bAvg_FTCC $=$ conj $($ F_bAvg_FT $)$;

F_bAvg_FFT=F_bAvg_FTCC. $*$ F_bAvg_FT;

$\%$ compute FFT's of avg + stdev S-L interface frame-by-frame for $i=1: N F$;

F_aStd_FT $(:, \dot{i})=f f t\left(F \_a S t d(:, i)\right)$;

end;

F_aStd_FTCC $=\operatorname{conj}\left(F_{-}\right.$aStd_FT);

F_aStd_FFT $=$ F_aStd_FTCC. $*$ F_aStd_FT;

$\%$ compute FFT's of avg - stdev S-L interface frame-by-frame for $i=1: N F$;

F_bStd_FT $(:, i)=f f t\left(F \_b S t d(:, i)\right)$; 
end ;

F_bStd_FTCC $=$ conj $\left(F_{-} b S t d \_F T\right)$;

F_bStd_FFT $=F \_b S t d \_F T C C . * F \_b S t d \_F T$;

\%concatenate all measurements into a single array, FFT it PH_all=reshape $(\mathrm{PH}, \mathrm{R} * \mathrm{C}, 1)$;

PH_all_FT $=f f t\left(P H \_a l l\right)$;

PH_all_FTCC=conj $\left(\mathrm{PH}_{-}\right.$all_FT $)$;

PH_all_FFT $=$ PH_all_FT. $*$ PH_all_FTCC;

toc 


\section{Bibliography}

[1] S Arai, S Tsukimoto, H Miyai, and H Saka. High-resolution in-situ transmission electron microscopy observation of a solid-liquid interface in the al-si system. Journal of Electron microscopy, 48(4):317-321, 1999.

[2] James M Howe. Direct observation of order in the liquid at a solid-liquid interface by high-resolution transmission electron microscopy. Philosophical Magazine A, 74(3):761-775, 1996.

[3] SH Oh, Y Kauffmann, C Scheu, WD Kaplan, and M Rühle. Ordered liquid aluminum at the interface with sapphire. Science, 310(5748):661-663, 2005.

[4] John Desmond Bernal. The bakerian lecture, 1962. the structure of liquids. Proceedings of the Royal Society of London. Series A, Mathematical and Physical Sciences, pages 299-322, 1964.

[5] Wayne D Kaplan and Yaron Kauffmann. Structural order in liquids induced by interfaces with crystals. Annu. Rev. Mater. Res., 36:1-48, 2006.

[6] James M Howe. Interfaces in materials. 1997.

[7] D Turnbull and J Prins. Physics of non-crystalline solids. In Proceedings Int. Conf. at Delft (July 1964), North-Holland Publ, Amsterdam, page 41, 1965.

[8] Frans Spaepen. A structural model for the solid-liquid interface in monatomic systems. Acta Metallurgica, 23(6):729-743, 1975.

[9] A Bonissent and B Mutaftschiev. A computer built random model for simulation of the crystal-melt interface. Philosophical Magazine, 35(1): 65-73, 1977. 
[10] H Ramalingam, M Asta, A Van de Walle, and JJ Hoyt. Atomic-scale simulation study of equilibrium solute adsorption at alloy solid-liquid interfaces. Interface Science, 10(2-3):149-158, 2002.

[11] Adham Hashibon, Joan Adler, Michael W. Finnis, and Wayne D. Kaplan. Atomistic study of structural correlations at a liquid-solid interface. Computational Materials Science, 24(4):443 - 452, 2002. ISSN 0927-0256. doi: http://dx.doi.org/10.1016/S0927-0256(01)00265-8. URL http: //www.sciencedirect.com/science/article/pii/S0927025601002658.

[12] H Men and Z Fan. Molecular dynamic simulation of the atomic structure of aluminum solid-liquid interfaces. Materials Research Express, 1(2):025705, 2014. URL http://stacks.iop.org/2053-1591/1/i=2/a=025705.

[13] S Tsukimoto, S Arai, and H Saka. High-resolution in situ electron microscopy of a silicon surface modification by molten aluminium at high temperatures. Philosophical magazine letters, 79(12):913-918, 1999.

[14] S Tsukimoto, S Arai, M Konno, T Kamino, K Sasaki, and H Saka. In situ high resolution electron microscopy/electron energy loss spectroscopy observation of wetting of a si surface by molten al. Journal of microscopy, 203(1):17-21, 2001.

[15] K Sasaki and H Saka. In situ high-resolution electron microscopy observation of the melting process of in particles embedded in an al matrix. Philosophical Magazine A, 63(6):1207-1220, 1991.

[16] Maria Gandman, Yaron Kauffmann, Christoph T Koch, and Wayne D Kaplan. Direct quantification of ordering at a solid-liquid interface using aberration corrected transmission electron microscopy. Physical review letters, 110(8):086106, 2013.

[17] Frans Spaepen. Homogeneous nucleation and the temperature dependence of the crystal-melt interfacial tension. Solid State Physics, 47:1-32, 1994. 
[18] JQ Broughton and GH Gilmer. Molecular dynamics investigation of the crystal-fluid interface. vi. excess surface free energies of crystal-liquid systems. The Journal of chemical physics, 84(10):5759-5768, 1986.

[19] Ruslan L. Davidchack and Brian B. Laird. Direct calculation of the hard-sphere crystal /melt interfacial free energy. Phys. Rev. Lett., 85: 4751-4754, Nov 2000. doi: 10.1103/PhysRevLett.85.4751. URL http://link.aps.org/doi/10.1103/PhysRevLett.85.4751.

[20] J. J. Hoyt, Mark Asta, and Alain Karma. Method for computing the anisotropy of the solid-liquid interfacial free energy. Phys. Rev. Lett., 86: 5530-5533, Jun 2001. doi: 10.1103/PhysRevLett.86.5530. URL http://link.aps.org/doi/10.1103/PhysRevLett.86.5530.

[21] James R. Morris. Complete mapping of the anisotropic free energy of the crystal-melt interface in al. Phys. Rev. B, 66:144104, Oct 2002. doi: 10.1103/PhysRevB.66.144104. URL http://link.aps.org/doi/10.1103/PhysRevB.66.144104.

[22] Ruslan L. Davidchack, James R. Morris, and Brian B. Laird. The anisotropic hard-sphere crystal-melt interfacial free energy from fluctuations. The Journal of Chemical Physics, 125(9):094710, 2006. doi: http://dx.doi.org/10.1063/1.2338303. URL http://scitation.aip.org/ content/aip/journal/jcp/125/9/10.1063/1.2338303.

[23] F Ercolessi and J. B Adams. Interatomic potentials from first-principles calculations: The force-matching method. Europhys. Lett., 26(8):583-588, jun 1994. doi: 10.1209/0295-5075/26/8/005. URL http://dx.doi.org/10.1209/0295-5075/26/8/005.

[24] M. Asta, J. J. Hoyt, and A. Karma. Calculation of alloy solid-liquid interfacial free energies from atomic-scale simulations. Phys. Rev. B, 66: 100101, Sep 2002. doi: 10.1103/PhysRevB.66.100101. URL http://link.aps.org/doi/10.1103/PhysRevB.66.100101. 
[25] H. Ramalingam, M. Asta, A. van de Walle, and J.J. Hoyt. Atomic-scale simulation study of equilibrium solute adsorption at alloy solid-liquid interfaces. Interface Science, 10(2-3):149-158, 2002. ISSN 0927-7056. doi: 10.1023/A:1015889313170. URL http://dx.doi.org/10.1023/A\%3A1015889313170.

[26] K. L. Merkle, L. J. Thompson, and Fritz Phillipp. Collective effects in grain boundary migration. Phys. Rev. Lett., 88:225501, May 2002. doi: 10.1103/PhysRevLett.88.225501. URL http://link.aps.org/doi/10.1103/PhysRevLett.88.225501.

[27] K.L. Merkle, L.J. Thompson, and F. Phillipp. In-situ hrem studies of grain boundary migration. Interface Science, 12(2-3):277-292, 2004. ISSN 0927-7056. doi: 10.1023/B:INTS.0000028657.72527.5b. URL http://dx.doi.org/10.1023/B\%3AINTS . $0000028657.72527 .5 b$.

[28] Nastaran Raffler and James M Howe. A high-resolution time-resolved study of incoherent interface motion during the massive transformation in tial alloy. Metallurgical and Materials Transactions A, 37(3):873-878, 2006.

[29] C Laird and HI Aaronson. The growth of $\gamma$ plates in an al-15\% ag alloy. Acta Metallurgica, 17(4):505-519, 1969.

[30] James M Howe. Atomic structure, composition, mechanisms and dynamics of transformation interfaces in diffusional phase transformations. Materials transactions-JIM, 39(1):3-23, 1998.

[31] R N Clough, G Moldovan, and A I Kirkland. Direct detectors for electron microscopy. J. Phys.: Conf. Ser., 522:012046, jun 2014. doi: 10.1088/1742-6596/522/1/012046. URL http://dx.doi.org/10.1088/1742-6596/522/1/012046.

[32] T Radetic, A Gautam, C Ophus, C Czarnik, and U Dahmen. High resolution observations of interface dynamics using a direct electron detection camera. Microsc. Microanal, 20:3, 2014. 
[33] James M Howe and Hiroyasu Saka. In situ transmission electron microscopy studies of the solid-liquid interface. MRS bulletin, 29(12):951-957, 2004.

[34] GA Storaska and JM Howe. In-situ transmission electron microscopy investigation of surface-oxide, stress-relief mechanisms during melting of sub-micrometer al-si alloy particles. Materials Science and Engineering: A, 368(1):183-190, 2004.

[35] Santhana K Eswaramoorthy, James M Howe, and Govindarajan Muralidharan. In situ determination of the nanoscale chemistry and behavior of solid-liquid systems. Science, 318(5855):1437-1440, 2007.

[36] Santhana K Eswara Moorthy and James M Howe. Kinetic analyses of the growth and dissolution phenomena of primary si and $\alpha$-al in partially molten al-si (-cu-mg) alloy particles using in situ transmission electron microscopy. Metallurgical and Materials Transactions A, 42(6):1667-1674, 2011.

[37] T. Yokota, J. M. Howe, W. A. Jesser, and M. Murayama. Fractional brownian motion of an al nanosphere in liquid al-si alloy under electron-beam irradiation. Journal of Applied Physics, 95(10), 2004.

[38] Justin D Starr and James M Howe. Investigation of the effects of in situ heating and cooling of al-si nanoparticles using the transmission electron microscope. Undergraduate Thesis, University of Virginia, 2009.

[39] CC Ahn and OL Krivanek. EELS atlas. Gatan, 1983.

[40] JL Murray and AJ McAlister. The al-si (aluminum-silicon) system. Bulletin of Alloy Phase Diagrams, 5(1):74-84, 1984.

[41] Ffmpeg. https://www.ffmpeg.org/. Accessed: 2014-10-13.

[42] Fiji is just imagej. http://http://fiji.sc/Fiji. Accessed: 2014-06-30.

[43] Qingzong Tseng. Template matching and slice alignment. https://sites. google.com/site/qingzongtseng/template-matching-ij-plugin. 
[44] Pierre Villars, A Prince, H Okamoto, et al. Handbook of ternary alloy phase diagrams, volume 2. ASM international, 1995.

[45] Prakash Palanisamy and James M Howe. In situ observation of cu segregation and phase nucleation at a solid-liquid interface in an al alloy. Acta Materialia, 61(12):4339-4346, 2013.

[46] Jarek Dabrowski and Hans-Joachim Müssig. Silicon Surfaces and Formation of interfaces: basic science in the industrial world. World Scientific, 1999.

[47] PM Kelly and M-X Zhang. Edge-to-edge matching - the fundamentals. Metallurgical and Materials Transactions A, 37(3):833-839, 2006.

[48] GJ Shiflet and JH van der Merwe. The role of structural ledges as misfit-compensating defects: fcc-bcc interphase boundaries. Metallurgical and Materials Transactions A, 25(9):1895-1903, 1994.

[49] Abhay Raj S Gautam and James M Howe. A method to predict the orientation relationship, interface planes and morphology between a crystalline precipitate and matrix. part i. approach. Philosophical Magazine, 91(24):3203-3227, 2011.

[50] JW Matthews. Epitaxial growth. Elsevier, 2012.

[51] AA Baski, SC Erwin, and LJ Whitman. The structure of silicon surfaces from (001) to (111). Surface science, 392(1):69-85, 1997.

[52] Charles Kittel. Introduction to solid state physics. 2005, 2005. 